\title{
Hypernuclei and massive neutron stars
}

\author{
M. Fortin \\ N. Copernicus Astronomical Center, Polish Academy of Sciences, Bartycka 18, 00-716 Warszawa, Poland
}

S. S. Avancini

Departamento de Física, CFM, Universidade Federal de Santa Catarina Florianópolis, SC, CP. 476, CEP 88.040-900, Brazil

C. Providência and I. Vidaña

CFisUC, Department of Physics, University of Coimbra, 3004-516 Coimbra, Portugal

(Received 23 January 2017; published 14 June 2017)

\begin{abstract}
Background: The recent accurate measurement of the mass of two pulsars close to or above $2 \mathrm{M}_{\odot}$ has raised the question of whether such large pulsar masses allow for the existence of exotic degrees of freedom, such as hyperons, inside neutron stars.

Purpose: In the present work, we will investigate, within a phenomenological relativistic mean field approach, how the existing hypernuclei properties may constrain the neutron star equation of state and confront the neutron star maximum masses obtained with equations of state calibrated to hypernuclei properties with the astrophysical $2 \mathrm{M}_{\odot}$ constraint.

Method: The study is performed using a relativistic mean field approach to describe both the hypernuclei and the neutron star equations of state. Unified equations of state are obtained. A set of five models that describe $2 \mathrm{M}_{\odot}$ when only nucleonic degrees of freedom are employed. Some of these models also satisfy other well-established laboratory or theoretical constraints.

Results: The $\Lambda$-meson couplings are determined for all the models considered, and the $\Lambda$ potential in symmetric nuclear matter and $\Lambda$ matter at saturation are calculated. Maximum neutron star masses are determined for two values of the $\Lambda-\omega$ meson coupling, $g_{\omega \Lambda}=2 g_{\omega N} / 3$ and $g_{\omega \Lambda}=g_{\omega N}$, and a wide range of values for $g_{\phi \Lambda}$. Hyperonic stars with the complete baryonic octet are studied, restricting the coupling of the $\Sigma$ and $\Xi$ hyperons to the $\omega, \rho$, and $\sigma$ mesons due to the lack of experimental data, and maximum star masses calculated.

Conclusions: We conclude that, within a phenomenological relativistic mean field approach, the currently available hypernuclei experimental data and the lack of constraints on the asymmetric equation of state of nuclear matter at high densities set only a limited number of constraints on the neutron star matter equation of state using the recent $2 \mathrm{M}_{\odot}$ observations. It is shown that the $\Lambda$ potential in symmetric nuclear matter takes a value of $\sim 30-32 \mathrm{MeV}$ at saturation for the $g_{\omega \Lambda}$ coupling given by the SU(6) symmetry, being of the order of the values generally used in the literature. On the other hand, the $\Lambda$ potential in $\Lambda$ matter varies between -14 and $-8 \mathrm{MeV}$, taking for vector mesons couplings the $\mathrm{SU}(6)$ values, at variance with generally employed values between -1 and $-5 \mathrm{MeV}$. If the $\mathrm{SU}(6)$ constraint is relaxed and the vector meson couplings to hyperons are kept to values not larger than those of nucleons, then values between -13 and $+9 \mathrm{MeV}$ are obtained.
\end{abstract}

DOI: 10.1103/PhysRevC.95.065803

\section{INTRODUCTION}

Neutron stars are among the smallest and densest objects in the Universe. With radii of the order of $\sim 10 \mathrm{~km}$ and masses that can be at least as large as two solar masses, matter inside neutron stars is subject to extreme conditions of density, isospin asymmetry, and magnetic field intensities. These objects constitute perfect laboratories to study nuclear matter under extreme conditions and the QCD phase diagram at low temperatures and high densities, and therefore they have been attracting attention in different fields of physics. Traditionally neutron star matter has been modeled as a uniform neutron-rich fluid in equilibrium with respect to the weak interactions ( $\beta$-stable matter) surrounded by a nonhomogeneous crust. Neutrons in the inner crust and neutrons and protons in the uniform core of the star are expected to be superfluid. Because of the large value of the density, new degrees of freedom are expected to appear in the inner core of neutron stars in addition to nucleons. Among others, hyperons, Bose-Einstein condensates of kaons or pions, and even deconfined quark matter have been considered.

Unlike in terrestrial conditions, where hyperons are unstable and decay into nucleons through weak interactions, matter in neutron stars maintains the weak equilibrium between the decays and their inverse capture processes. Since the pioneering work of Ambartsumyan and Saakyan in 1960 [1], the presence of hyperons in neutron stars has been studied by many authors using either microscopic [2-6] or phenomenological $[7,8]$ approaches to the neutron star matter equation of state (EoS). All these works agree that hyperons may appear in the inner core of neutron stars at densities around $\sim(2-3) \times n_{0} \quad\left(n_{0}=0.16 \mathrm{fm}^{-3}\right)$, when the nucleon chemical potential is large enough to make the conversion of a nucleon into a hyperon energetically favorable. This conversion relieves the Fermi pressure exerted by nucleons, making the EoS softer. Consequently, the mass of the star, and, in particular, its maximum value $M_{\max }$, is substantially 
reduced. In microscopic calculations (see, e.g., Refs. [2,3]), this reduction can be even below the value of the mass of the Hulse-Taylor pulsar $\left(1.4408 \pm 0.0003 \mathrm{M}_{\odot}\right)$ [9]. This is not the case, however, in phenomenological calculations which find values of $M_{\max }$ compatible with the canonical value above. In fact, most relativistic mean field (RMF) models including hyperons predict maximum masses in the range $1.4-1.8 \mathrm{M}_{\odot}$ [7], although with some parametrizations masses as large as $2 \mathrm{M}_{\odot}$ could be even obtained [10,11].

The presence of hyperons in neutron stars seems to be energetically unavoidable, although the strong softening of the EoS associated with their appearance (notably in microscopic models) leads to the prediction of maximum masses not compatible with observations. A natural question, therefore, arises: Can hyperons still be present in the interior of neutron stars if $M_{\max }$ is reduced to values not compatible with astrophysical observations, although their presence is energetically favorable? This question is at the origin of what has been called the hyperon puzzle. Its nontrivial solution is currently a subject of intense research, especially in view of the recent measurements of unusually high masses of the millisecond pulsars PSR J1614-2230 $\left(1.928 \pm 0.017 \mathrm{M}_{\odot}\right)$ $[12,13]$ and PSR J0348+0432 (2.01 $\left.\pm 0.04 \mathrm{M}_{\odot}\right)$ [14], which ruled out almost all currently proposed EoS with hyperons. The solution of this problem demands a mechanism that could eventually provide the additional repulsion to make the EoS stiffer and the maximum mass compatible with observation [15]. Three different mechanisms have been proposed: (i) the inclusion of a repulsive hyperon-hyperon interaction through the exchange of vector mesons [16-19], or less attractive scalar $\sigma$-meson exchange [20], at the cost of potentially making the EoS too stiff around and below saturation density and therefore incompatible with recent quantum Monte Carlo nuclear matter [21] and chiral effective field theory [22] calculations; (ii) the inclusion of repulsive hyperonic three-body forces [23-26]; or (iii) the possibility of a phase transition to deconfined quark matter at densities below the hyperon threshold [27-31]. An alternative way to circumvent the hyperon puzzle by invoking the appearance of other hadronic degrees of freedom such as, for instance, the $\Delta$ isobar that pushes the onset of hyperons to higher densities has also been considered [32]. We note that very recently Haidenbauer et al. [33] have shown that the $\Lambda$ single-particle potential, obtained in a Brueckner-HartreeFock calculation using a hyperon-nucleon interaction derived from an SU(3) chiral effective field theory, becomes strongly repulsive for densities larger than $2 n_{0}$, therefore shifting the onset of hyperons to extremely high densities and potentially solving the hyperon puzzle without the necessity of invoking any of these more exotic mechanisms.

In addition to the observation of massive neutron stars, more astrophysical constraints on the neutron star EoS and consequently on its hyperon content such as the measurement of their radius, moment of inertia, or the surface gravitational red shift from spectral lines may come in the near future thanks to the next generation of x-ray telescopes and radio observatories. No measurement of the two latter quantities has been obtained so far. Many techniques have been devised to determine neutron star radii but current estimates are still controversial both on theoretical and observational grounds (see, e.g., discussions in $[15,34,35]$. Some of the estimates predict radii of $\sim 10 \mathrm{~km}$ or less [36,37]. If this is confirmed by further analysis, then the simultaneous existence of massive neutron stars and objects with small radii would be a very complicated problem to solve for any of the existing models of the pure nucleonic EoS. A solution to this problem that has been proposed is the possible existence of the so-called "twin stars," stars with similar masses but smaller radii than those made only of nucleons. Recently, it has been conjectured that these twin stars could in fact be composed of strange hadronic or quark matter [38,39]. Future X-ray missions such as NICER [40], Athena [41], and potential LOFT-like missions [42] promise simultaneous determinations of the mass and radius with $\sim 5 \%$ precision.

In the present work, we explore the possibility of obtaining two solar mass hyperonic stars within the relativistic mean field (RMF) approach when the hyperon-meson couplings are constrained by the existing experimental hypernuclear data. We shall consider a set of models that satisfy the two solar mass constraint imposed by the pulsars J1614-2230 and J0348+0432 when only purely nucleonic degrees of freedom are considered and discuss the consequences of including hyperons when hypernuclear data are used to constrain the hyperon-nucleon and the hyperon-hyperon interactions. In particular, the experimental data on single and double $\Lambda$ hypernuclei will be taken into account in the model within the framework of the RMF approach, as done in Ref. [43]. Recently, a study with a similar objective has been performed in Ref. [20]. The authors of this work used symmetry arguments to fix the couplings of the vector mesons to hyperons and single $\Lambda$ hypernuclei binding energies to constrain the coupling of the $\sigma$ meson to the $\Lambda$ hyperon. The coupling of the other hyperons to the $\sigma$ meson were obtained, requiring that the lower bound on the maximum mass of the star be $2 \mathrm{M}_{\odot}$. In this work, we follow the same procedure to fix the $\Lambda-\sigma$ meson coupling, but a different approach is used for the other couplings. In particular, we take also into account the experimental data on double $\Lambda$ hypernuclei. A comparison between the two approaches is presented.

The paper is organized in the following way: In Sec. II, we describe and summarize the main properties of the different RMF parametrizations used in this work. Then, in Sec. III, we review the current status of available hypernuclear experimental data. In Sec. IV, we explain how the binding energies of single and double $\Lambda$ hypernuclei are used to calibrate the coupling constants of the $\Lambda$ hyperon with the different mesons in the RMF models. We compare our results for the $\Lambda$ potential at saturation with the usual values taken in the literature and provide tables with values of the $\Lambda$ couplings calibrated to up-to-date hypernuclear data. Hyperonic and unified EOS are then built in Sec. V and their predictions for $M_{\max }$ are confronted with the existence of pulsars with $\mathbf{M}_{\odot}$. We finish by briefly summarizing our results and presenting our conclusions in Sec. VI.

\section{RMF MODELS}

In the following, five different RMF models, all predicting $2 \mathrm{M}_{\odot}$ purely nucleonic stars, are considered: four nonlinear 
TABLE I. Nuclear properties at saturation density $\left(n_{0}\right)$ predicted by the different RMF models used in this work: energy per nucleon $\left(E_{0}\right)$, compression modulus $(K)$, symmetry energy $(J)$, its slope $(L)$, and incompressibility $\left(K_{\text {sym }}\right)$ at the saturation point of uniform symmetric nuclear matter at the density $n_{0}$. The last column shows the value of neutron star maximum mass $\left(M_{\max }^{\mathrm{N}}\right)$ predicted by these models when only nucleonic degrees of freedom are considered.

\begin{tabular}{lccccrrrr}
\hline \hline Model & $\begin{array}{c}n_{0} \\
\left(\mathrm{fm}^{-3}\right)\end{array}$ & $\begin{array}{c}E_{0} \\
(\mathrm{MeV})\end{array}$ & $\begin{array}{c}K \\
(\mathrm{MeV})\end{array}$ & $\begin{array}{c}J \\
(\mathrm{MeV})\end{array}$ & $\begin{array}{c}L \\
(\mathrm{MeV})\end{array}$ & $\begin{array}{c}K_{\text {sym }} \\
(\mathrm{MeV})\end{array}$ & $\begin{array}{l}M_{\max }^{\mathrm{N}} \\
\left(\mathrm{M}_{\odot}\right)\end{array}$ & Ref. \\
\hline TM1 & 0.146 & -16.3 & 281.2 & 36.9 & 111.2 & 33.8 & 2.18 & {$[44]$} \\
TM2 $\omega \rho$ & 0.146 & -16.4 & 281.7 & 32.1 & 54.8 & -70.5 & 2.25 & {$[45]$} \\
NL3 & 0.149 & -16.2 & 271.6 & 37.4 & 118.9 & 101.6 & 2.77 & {$[46]$} \\
NL3 $\omega \rho$ & 0.148 & -16.2 & 271.6 & 31.7 & 55.5 & -7.6 & 2.75 & {$[47]$} \\
DDME2 & 0.152 & -16.1 & 250.9 & 32.3 & 51.2 & -87.1 & 2.48 & {$[48]$} \\
\hline \hline
\end{tabular}

Walecka type models with constant coupling parameters and one density-dependent model with coupling parameters that depend on the density. Among the former, we consider the parametrizations TM1 [44], TM2 $\omega \rho$ [45], NL3 [46], and NL3 $\omega \rho$ [47], and for the latter we choose the model DDME2 [48]. Some of their nuclear properties as well as their prediction for the neutron star maximum mass are presented in Table I. In the following, we briefly explain the reasons for the choice of these particular models.

The parametrization TM1 [44] was used in Ref. [43] to describe single and double $\Lambda$ hypernuclei, and we will consider it as a reference. This model includes a nonlinear $\omega$ meson term which softens the EoS at high densities and is the underlying model of the Shen-Toki-Oyamatzu-Sumiyoshi supernova EoS [49,50]. However, this EoS has a symmetry energy slope parameter that is too large $(L=110 \mathrm{MeV})[51]$ and does not satisfy the subsaturation neutron matter constraints imposed by microscopic calculations [22]. Including a nonlinear term that mixes the $\omega$ and $\rho$ mesons allows us to overcome these two shortcomings. This term has been added to the TM1 parametrization, resulting in the parametrization TM $2 \omega \rho$ [45] that has a weaker nonlinear $\omega$ term, making the EoS stiffer than TM1 at large densities. We also consider the NL3 parametrization [46], which was fitted to the ground-state properties of both stable and unstable nuclei. This parametrization predicts very large, purely nucleonic neutron star maximum masses but has the drawback of having, as TM1, a symmetry energy slope that is too large ( $L=118 \mathrm{MeV}$ ) [51]. Thus, we will also consider the parametrization NL3 $\omega \rho$ [47] with a softer density dependence of the symmetry energy due to inclusion of the nonlinear $\omega \rho$ term. We note here that in Ref. [52] this parametrization was one of only four parametrizations chosen as satisfying a set of generally accepted constraints and were still able to describe $2 \mathrm{M}_{\odot}$ stars. The model DDME2 with density-dependent couplings was another one of these four parametrizations, which we also choose in the present study. We note also that of these five parametrizations only TM1, TM2 $\omega \rho$, and DDME2 satisfy the constraints imposed by the flow of matter in heavy ion collisions [53] (see the discussion in Ref. [54]). However, since the analysis of the experimental flow data is quite complex and not totally model independent, this constraint
TABLE II. Parameter sets used in this work. The DDME2 parameters are defined at saturation density and the meson masses are given in $\mathrm{MeV}$.

\begin{tabular}{lccccc}
\hline \hline & TM1 & TM2 $\omega$ & NL3 & NL3 $\omega \rho$ & DDME2 \\
\hline$m_{\sigma}$ & 511.198 & 511.198 & 508.194 & 508.194 & 550.1238 \\
$m_{\omega}$ & 783 & 783 & 782.501 & 782.501 & 783 \\
$m_{\rho}$ & 770 & 770 & 763 & 763 & 763 \\
$g_{\sigma}$ & 10.029 & 9.998 & 10.217 & 10.217 & 10.5396 \\
$g_{\omega}$ & 12.614 & 12.503 & 12.868 & 12.868 & 13.0189 \\
$g_{\rho}$ & 9.264 & 11.303 & 8.948 & 11.277 & 7.3672 \\
$\kappa / M$ & 3.043 & 3.523 & 10.431 & 10.431 & 0 \\
$\lambda$ & 3.710 & -47.362 & -28.885 & -28.885 & 0 \\
$\xi$ & 0.0169 & 0.0113 & 0 & 0 & 0 \\
$\Lambda_{\mathrm{v}}$ & 0 & 0.03 & 0 & 0.03 & 0 \\
\hline \hline
\end{tabular}

should be taken with care. Therefore, we will also consider the two parametrizations NL3 and NL3 $\omega \rho$. The set of parameters of all the models is shown in Table II. The parameters for the DDME2 model are shown at saturation density.

The inclusion of hyperons in RMF models is performed in a quite natural way [55,56]. The hyperon-nucleon $(Y N)$ interaction is described by means of the exchange of $\sigma, \omega$, and $\rho$ mesons, similar to the nucleon-nucleon $(N N)$ one. The hyperon-hyperon $(Y Y)$ interaction is included in our model by considering also the coupling of hyperons with the hidden strangeness mesons $\sigma^{*}$ and $\phi$. The Lagrangian density for a system that includes the eight lightest baryons, i.e., the nucleon doublet (neutron $n$ and proton $p$ ) and the six lightest hyperons ( $\Lambda$, the $\Sigma^{+}, \Sigma^{0}, \Sigma^{-}$triplet, and the $\Xi^{0}, \Xi^{-}$doublet), reads $[44,45]$ as

$$
\begin{aligned}
\mathcal{L}= & \sum_{B} \bar{\Psi}_{B}\left[\gamma_{\mu} D_{B}^{\mu}-m_{B}^{*}\right] \Psi_{B} \\
& +\sum_{l=e, \mu} \bar{\psi}_{l}\left[i \gamma_{\mu} \partial^{\mu}-m_{l}\right] \psi_{l} \\
& +\frac{1}{2}\left(\partial_{\mu} \sigma \partial^{\mu} \sigma-m_{\sigma}^{2} \sigma^{2}\right)-\frac{1}{3 !} k \sigma^{3}-\frac{1}{4 !} \lambda \sigma^{4} \\
& +\frac{1}{2} m_{\omega}^{2} \omega_{\mu} \omega^{\mu}-\frac{1}{4} \Omega_{\mu \nu} \Omega^{\mu \nu}+\frac{1}{4 !} \xi g_{\omega}^{4}\left(\omega_{\mu} \omega^{\mu}\right)^{2} \\
& +\frac{1}{2} m_{\rho}^{2} \boldsymbol{\rho}_{\mu} \cdot \boldsymbol{\rho}^{\mu}-\frac{1}{4} \mathbf{P}_{\mu \nu} \cdot \mathbf{P}^{\mu \nu} \\
& +\Lambda_{\omega}\left(g_{\omega}^{2} \omega_{\mu} \omega^{\mu}\right)\left(g_{\rho}^{2} \boldsymbol{\rho}_{\mu} \cdot \boldsymbol{\rho}^{\mu}\right) \\
& +\frac{1}{2}\left(\partial_{\mu} \sigma^{*} \partial^{\mu} \sigma^{*}-m_{\sigma^{*}}^{2} \sigma^{* 2}\right) \\
& +\frac{1}{2} m_{\phi}^{2} \phi_{\mu} \phi^{\mu}-\frac{1}{4} \Phi_{\mu \nu} \Phi^{\mu \nu},
\end{aligned}
$$

where $D_{B}^{\mu}=i \partial^{\mu}-g_{\omega B} \omega^{\mu}-g_{\phi B} \phi^{\mu}-g_{\rho B} \boldsymbol{\tau}_{B} \cdot \boldsymbol{\rho}^{\mu}$ and $m_{B}^{*}=$ $m_{B}-g_{\sigma B} \sigma-g_{\sigma^{*} B} \sigma^{*}$ is the effective mass of baryon $B . \Psi_{B}$ and $\psi_{l}$ are the baryon and lepton Dirac fields, respectively, and $g_{i B}$ is the coupling constant of meson $i$ with baryon $B$. The mass of baryon $B$ and lepton $l$ are denoted by $m_{B}$ and $m_{l}$, respectively. The constants $k, \lambda$, and $\Lambda_{\omega}$ are the couplings associated with the nonlinear interaction terms, 
and $\boldsymbol{\tau}_{B}$ is the isospin operator. The mesonic field tensors are given by their usual expressions: $\Omega_{\mu \nu}=\partial_{\mu} \omega_{\nu}-\partial_{\nu} \omega_{\mu}, \boldsymbol{P}_{\mu \nu}=$ $\partial_{\mu} \boldsymbol{\rho}_{v}-\partial_{\nu} \boldsymbol{\rho}_{\mu}-g_{\rho}\left(\boldsymbol{\rho}_{\mu} \times \boldsymbol{\rho}_{\nu}\right)$, and $\Phi_{\mu \nu}=\partial_{\mu} \phi_{v}-\partial_{\nu} \phi_{\mu}$. The couplings $g_{i, B}$ are constant for the models TM1, TM2 $\omega \rho$, NL3, and NL3 $\omega \rho$, whereas they are density dependent in DDME2. We will explain later in Sec. IV how all these couplings are fixed. Here, we simply indicate that the coupling constants of the nucleons with the $\sigma^{*}$ and $\phi$ mesons are set to zero.

\section{BRIEF OVERVIEW OF HYPERNUCLEAR PHYSICS}

Whereas the $N N$ interaction is fairly well known due to the large number of existing scattering data, the $Y N$ and $Y Y$ ones are still poorly constrained. Experimental difficulties due to the short lifetime of hyperons and the low-intensity beam fluxes have limited the number of $\Lambda N$ and $\Sigma N$ scattering events to several hundred [57-61] and that of $\Xi N$ events to very few. In the case of the $Y Y$ interaction, the situation is even worse because no scattering data exist at all. This limited amount of data is not enough to fully constrain these interactions.

In the absence of scattering data, alternative information on the $Y N$ and $Y Y$ interactions can be obtained from the study of hypernuclei, bound systems composed of nucleons and one or more hyperons. Hypernuclei were discovered in 1952 with the observation of a hyperfragment in a balloon-flown emulsion stack by Danysz and Pniewski [62]. Since then, more than 40 single $\Lambda$ hypernuclei, and few double $\Lambda$ [63-70] and single $\Xi[71,72]$ ones have been identified by the use of high-energy accelerators and modern electronic counters. However, it has not been possible to prove without any ambiguity the existence of $\Sigma$ hypernuclei (see, e.g., Refs. [73-81]), which suggests that the $\Sigma$ nucleon interaction is most probably repulsive [82-91].

Single $\Lambda$ hypernuclei can be produced by several mechanisms, such as $\left(K^{-}, \pi^{-}\right)$strangeness exchange reactions, where a neutron hit by a $K^{-}$is changed into a $\Lambda$ emitting a $\pi^{-}$. The analysis of these reactions showed many of the hypernuclear characteristics such as, for instance, the small spin-orbit strength of the $Y N$ interaction, or the fact that the $\Lambda$ essentially retains its identity inside the nucleus. The use of $\pi^{+}$ beams permitted $\left(\pi^{+}, K^{+}\right)$associated production reactions, where an $s \bar{s}$ pair is created from the vacuum, and a $K^{+}$and a $\Lambda$ are produced in the final state. The electroproduction of hypernuclei by means of the reaction $\left(e, e^{\prime} K^{+}\right)$provides a high-precision tool for the study of hypernuclear spectroscopy [92] due to the excellent spatial and energy resolution of the electron beams. Recently, the HypHI Collaboration at FAIR/GSI has proposed a new way to produce hypernuclei by using stable and unstable heavy ion beams [93]. The $\Lambda$, and the ${ }_{\Lambda}^{3} \mathrm{H}$ and ${ }_{\Lambda}^{4} \mathrm{H}$ hypernuclei have been observed in a first experiment performed using a ${ }^{6} \mathrm{Li}$ beam on a ${ }^{12} \mathrm{C}$ target at $2 \mathrm{~A}$ GeV [94].

Hypernuclei can be produced in excited states if a nucleon in a $p$ or higher shell is replaced by a hyperon. The energy of these excited states can be released either by emitting nucleons, or sometimes when the hyperon moves to lower energy states, by the emission of $\gamma$ rays. Measurements of $\gamma$-ray transitions in $\Lambda$ hypernuclei has allowed the analysis of the excited levels with an excellent energy resolution. Systematic studies of single $\Lambda$ hypernuclei indicate that the $\Lambda \mathrm{N}$ interaction is clearly attractive [95].

$\Sigma$ hypernuclei can also be produced by the mechanisms just described. However, as said before, there is not yet an unambiguous experimental confirmation of their existence.

To produce double- $\Lambda$ hypernuclei, first it is necessary to create a $\Xi^{-}$through reactions like

$$
K^{-}+p \rightarrow \Xi^{-}+K^{+}
$$

or

$$
p+\bar{p} \rightarrow \Xi^{-}+\bar{\Xi}^{+} .
$$

Then, the $\Xi^{-}$should be captured in an atomic orbit and interact with the nuclear core, producing two $\Lambda$ hyperons by means of the process

$$
\Xi^{-}+p \rightarrow \Lambda+\Lambda+28.5 \mathrm{MeV},
$$

providing about $30 \mathrm{MeV}$ of energy that is equally shared between the two $\Lambda$ 's in most cases, leading to the escape of one or both hyperons from the nucleus. $\Xi$ hypernuclei can be produced by means of the reactions (2) and (3) and, as said above, very few of them have been identified. The analysis of the experimental data from production reactions such as ${ }^{12} \mathrm{C}\left(K^{-}, K^{+}\right){ }_{\Xi^{-}}^{12} \mathrm{Be}[71]$ indicates an attractive $\Xi$-nucleus interaction of the order of about $-14 \mathrm{MeV}$. Here, we should mention the very recent observation of a deeply bound state of the $\Xi^{-}{ }^{14} \mathrm{~N}$ system with a binding energy of $4.38 \pm 0.25$ $\mathrm{MeV}$ by Nakazawa et al. [72]. This event provides the first clear evidence of a deeply bound state of this system by an attractive $\Xi N$ interaction. Future $\Xi$ hypernuclei experiments are being planned at J-PARC.

Double-strange hypernuclei are currently the best systems to investigate the properties of the baryon-baryon interaction in the strangeness $S=-2$ sector. The $\Lambda \Lambda$ bond energy $\Delta B_{\Lambda \Lambda}$ in double $\Lambda$ hypernuclei can be determined experimentally from the measurement of the binding energies of double and single $\Lambda$ hypernuclei as

$$
\Delta B_{\Lambda \Lambda}=B_{\Lambda \Lambda}\left(\begin{array}{l}
A \\
\Lambda \Lambda
\end{array}\right)-2 B_{\Lambda}\left(\begin{array}{l}
A-1 \\
\Lambda
\end{array}\right) .
$$

Emulsion experiments [64,66-68] have reported the formation of a few double $\Lambda$ hypernuclei: ${ }_{\Lambda \Lambda}^{6} \mathrm{He},{ }_{\Lambda \Lambda}^{10} \mathrm{Be}$, and ${ }_{\Lambda \Lambda}^{13} \mathrm{~B}$. From the subsequent analysis of these emulsion experiments, a quite large $\Lambda \Lambda$ bound energy of around 4 to $5 \mathrm{MeV}$ was deduced, contrary to expectation from SU(3) (Stoks and Rijken in Ref. [96]). We should also note that the identification of some of these double $\Lambda$ hypernuclei was ambiguous. Therefore, careful attention should be paid when using the data from this old analysis to put any kind of constraint on the $\Lambda \Lambda$ interaction. However, a new ${ }_{\Lambda \Lambda}^{6}$ He candidate having a $\Lambda \Lambda$ bond energy

$$
\Delta B_{\Lambda \Lambda}=1.01 \pm 0.2_{-0.11}^{+0.18} \mathrm{MeV}
$$

was unambiguously observed in 2001 at KEK [70]. This value has then been recently revised due to a change in the value of the $\Xi^{-}$mass [97]:

$$
\Delta B_{\Lambda \Lambda}=0.67 \pm 0.17 \mathrm{MeV} .
$$

In this work, we will use these two values of $\Delta B_{\Lambda \Lambda}$ to constrain the coupling of the $\Lambda$ hyperon with the $\sigma^{*}$ meson. 


\section{CALIBRATION OF THE $\Lambda$ MESON COUPLING CONSTANTS}

Since the $\Lambda$ is an isospin singlet, it does not couple with the $\rho$ meson. Therefore, only the coupling constants with the $\sigma, \omega, \sigma^{*}$, and $\phi$ mesons should be fixed. The usual procedure to fix these couplings consists in using the SU(6) symmetry to determine the couplings of the $\Lambda$ with the vector mesons in terms of those of the nucleons

$$
\begin{aligned}
& R_{\omega \Lambda}=g_{\omega \Lambda} / g_{\omega N}=2 / 3, \\
& R_{\phi \Lambda}=g_{\phi \Lambda} / g_{\omega N}=-\frac{\sqrt{2}}{3},
\end{aligned}
$$

and the $\Lambda$-scalar mesons ones by using data derived indirectly from hypernuclei. In particular, these couplings are obtained by imposing the value of the $\Lambda$ potential in symmetric nuclear matter, $U_{\Lambda}^{N}$, and the value of the $\Lambda$ potential in $\Lambda$ matter, $U_{\Lambda}^{\Lambda}$, at saturation, defined respectively as

$$
U_{\Lambda}^{N}\left(n_{0}\right)=-\left(g_{\sigma \Lambda}+g_{\sigma \Lambda}^{\prime} \rho_{s}\right) \sigma_{0}+\left(g_{\omega \Lambda}+g_{\omega \Lambda}^{\prime} n_{0}\right) \omega_{0}
$$

and

$$
\begin{aligned}
U_{\Lambda}^{\Lambda}\left(n_{0}\right)= & -\left(g_{\sigma \Lambda}+g_{\sigma \Lambda}^{\prime} \rho_{s}\right) \sigma_{0}-\left(g_{\sigma^{*} \Lambda}+g_{\sigma^{*} \Lambda}^{\prime} \rho_{s}\right) \sigma_{0}^{*} \\
& +\left(g_{\omega \Lambda}+g_{\omega \Lambda}^{\prime} n_{0}\right) \omega_{0}+\left(g_{\phi \Lambda}+g_{\phi \Lambda}^{\prime} n_{0}\right) \phi_{0}
\end{aligned}
$$

with $\sigma_{0}, \omega_{0}, \sigma_{0}^{*}$, and $\phi_{0}$ being the mean-field values of the $\sigma, \omega$, $\sigma^{*}$, and $\phi$ meson fields, respectively, and $\rho_{s}$ being the scalar density. The quantities $g_{i \Lambda}^{\prime}$ are the derivatives with respect to the density of the couplings $g_{i \Lambda}$ and are only different from zero for models with density-dependent couplings (see the discussion below). All quantities are calculated at the saturation density $n_{0}$. Values of $U_{\Lambda}^{N}\left(n_{0}\right) \simeq-30 \mathrm{MeV}$ and $U_{\Lambda}^{\Lambda}\left(n_{0}\right)=-5 \mathrm{MeV}$ are usually employed in the literature to determine these couplings. The first value results from the extrapolation at $A^{-2 / 3}=0$ of the experimental binding energy of single- $\Lambda$ hypernuclei, $A$ being the mass number of the hypernucleus. The second one is usually obtained from the identification $U_{\Lambda}^{\Lambda}\left(n_{0}\right)=-\Delta B_{\Lambda \Lambda}$ and the use of the value $\Delta B_{\Lambda \Lambda}=5 \mathrm{MeV}$ deduced from the emulsion experiments of Refs. [64,66-68] carried out in the 1960s and 1990s. However, as pointed before, due to the ambiguous identification of some of the double $\Lambda$ hypernuclei in these experiments, one has to be very careful when using these old experimental data.

In this work, however, we follow a different procedure. The couplings of the $\Lambda$ with the various mesons are calibrated by fitting the experimental binding energy of $\Lambda$ hypernuclei following the approach of Refs. [43,98]. Before we give more specific details on the calibration procedure, we should note that we have considered two different approaches to fix the hyperon-meson couplings in the case of the model DDME2. First, we use the experimental constraints and symmetry arguments to fix the magnitude of the couplings at saturation density, as done for the other models with constant couplings. Then we consider (i) that the hyperon-meson couplings do not depend on the density (this approach is designated simply as DDME2) and (ii) we assume for the hyperon couplings the same density dependence of the nucleonic couplings (this model will be referred as DDME2D). For explicit density dependence of the couplings, the interested reader is referred to Ref. [48].

Hypernuclei binding energies are obtained by solving the Dirac equations for the nucleons and the $\Lambda$ obtained from the Lagrangian density (1) using the method described in Refs. [99,100]. In this approach, the hypernucleus wave function is a Slater determinant and only the lowest single-particle positive-energy states are occupied. We use the relativistic mean field approximation where the meson field operators are replaced by their expectation values and negative-energy states are neglected (no-sea approximation). The numerical algorithm consists in the expansion of the Dirac spinors and mesonic fields in terms of the harmonic oscillator basis. Therefore, the Dirac and Klein-Gordon equations are transformed into matrix equations that are solved in a self-consistent way until convergence is achieved. For an accurate description of light hypernuclei, the center-of-mass correction, instead of the simple correction

$$
E_{\mathrm{COM}}=\frac{3}{4} 41 A^{-1 / 3} \quad[\mathrm{MeV}]
$$

commonly used in the literature [100], is calculated through the expression

$$
E_{\mathrm{COM}}=\frac{\left\langle P^{2}\right\rangle}{2 M},
$$

where $\left\langle P^{2}\right\rangle$ is the expectation value of the squared total momentum and $M$ is the hypernucleus total mass. The former expectation value is calculated from the actual many-body state of the hypernucleus.

As in Ref. [43], we include the tensor term

$$
\mathcal{L}_{T \Lambda}=\bar{\psi}_{\Lambda} \frac{f_{\omega \Lambda}}{2 M_{\Lambda}} \sigma^{\mu \nu} \partial_{\nu} \omega_{\mu} \psi_{\Lambda},
$$

which is important to get a weak $\Lambda$-nuclear spin-orbit interaction [101,102]. The spin-orbit potential for single $\Lambda$ hypernuclei is the result of two opposite contributions which partially cancel out; one is the usual associated to the difference between the derivative of the scalar $(\sigma)$ and vector $(\omega)$ central potentials and the other is due to the tensor term.

Although no experimental data are available for the spinorbit splitting of $\Lambda$ hypernuclei, taking into account the tensor term, within the quark model $\left(f_{\omega \Lambda}=-g_{\omega \Lambda}\right)$, causes an improvement on the quality of the overall calibration of the coupling constants.

\section{A. Single $\Lambda$ hypernuclei}

For a given value of $R_{\omega \Lambda}$, the ratio $R_{\sigma \Lambda}=g_{\sigma \Lambda} / g_{\sigma N}$ is calibrated to reproduce the binding energies $B_{\Lambda}$ of hypernuclei in the $s$ and $p$ shells (see Fig. 1). The experimental data used in the calibration are taken from Table IV of Ref. [103]. The best value of $R_{\sigma \Lambda}$ is determined by minimizing the function

$$
\chi^{2}=\frac{1}{N} \sum_{i=1}^{N}\left(\frac{B_{\Lambda_{i}}^{\text {exp }}-B_{\Lambda_{i}}^{\text {the }}}{B_{\Lambda_{i}}^{\exp }}\right)^{2},
$$




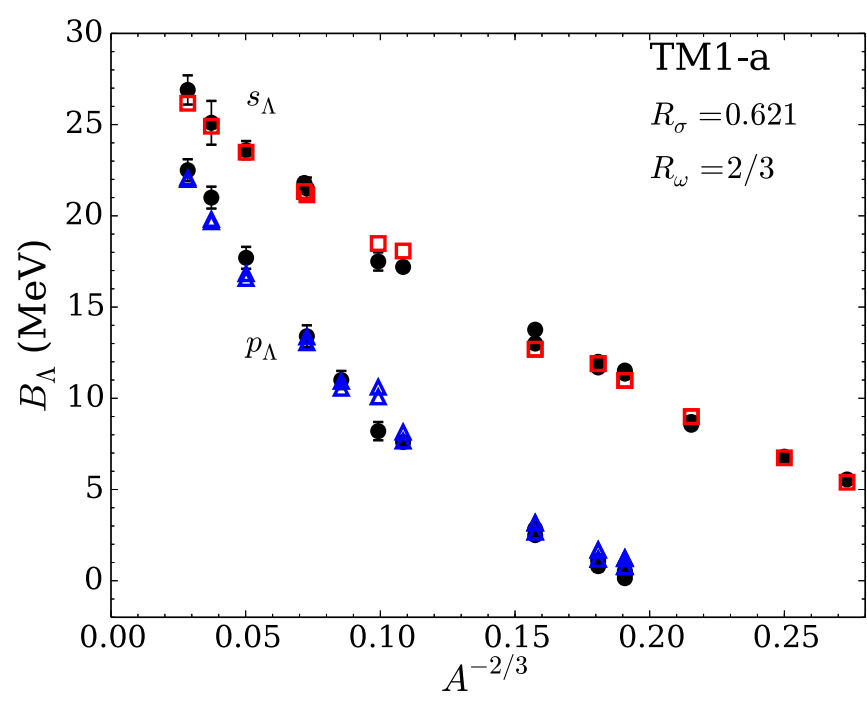

FIG. 1. For the TM1-a model, experimental values of the binding energies $B_{\Lambda}$ in the $s$ and $p$ shells of single $\Lambda$ hypernuclei (black circles) and modeled values (red squares and blue triangles, respectively) obtained after adjusting $R_{\sigma \Lambda}$ in order to minimize the quantity $\chi^{2}$ defined in Eq. (15). For $\Lambda$ in $p$ shells, $p_{1 / 2}$ and $p_{3 / 2}$ states are plotted.

where $B_{\Lambda i}^{\exp }$ and $B_{\Lambda i}^{\text {the }}$ are, respectively, the values of the binding energy of a given single $\Lambda$ hypernuclei $i$ obtained experimentally and from the modeling, and $N$ is the total number of $\Lambda$ hypernuclei for which experimental data are available. Equal, or very close, values of $R_{\sigma \Lambda}$ are obtained if only heavy hypernuclei with $Z>20$ are considered or if the denominator in Eq. (15) is replaced by the error bar on the experimental measurements of the binding energies. Similarly, the calibration is hardly affected if only $s$-shell binding energies are taken into account or if both shells are included. In Table III, we indicate, for two different values of $R_{\omega \Lambda}$, the calibrated values of $R_{\sigma \Lambda}$ as well as the associated

TABLE III. Calibration to single $\Lambda$ hypernuclei: For given $R_{\omega \Lambda}$, values of $R_{\sigma \Lambda}$ calibrated to reproduce the binding energies $B_{\Lambda}$ of hypernuclei in the $s$ and $p$ shells. The last column contains the value of the $\Lambda$ potential in symmetric baryonic matter at saturation in $\mathrm{MeV}$, for reference.

\begin{tabular}{lccc}
\hline \hline Model & $R_{\omega \Lambda}$ & $R_{\sigma \Lambda}$ & $U_{\Lambda}^{N}\left(n_{0}\right)$ \\
\hline TM1-a & $2 / 3$ & 0.621 & -30 \\
TM1-b & 1 & 0.892 & -31 \\
TM2 $\omega \rho-\mathrm{a}$ & $2 / 3$ & 0.621 & -30 \\
TM2 $\omega \rho-\mathrm{b}$ & 1 & 0.891 & -31 \\
NL3-a & $2 / 3$ & 0.622 & -31 \\
NL3-b & 1 & 0.894 & -32 \\
NL3 $\omega \rho-\mathrm{a}$ & $2 / 3$ & 0.622 & -31 \\
NL3 $\omega \rho-\mathrm{b}$ & 1 & 0.894 & -32 \\
DDME2-a & $2 / 3$ & 0.615 & -32 \\
DDME2-b & 1 & 0.891 & -35 \\
DDME2D-a & $2 / 3$ & 0.621 & -32 \\
DDME2D-b & 1 & 0.896 & -35 \\
\hline \hline
\end{tabular}

value of the $\Lambda$ potential in symmetric baryonic matter at saturation $U_{\Lambda}^{N}\left(n_{0}\right)$ obtained from Eq. (10), for all the models considered. In this table and in the following for each RMF parametrization we consider two values of the ratio $R_{\omega \Lambda}$, one $R_{\omega \Lambda}=2 / 3$ corresponding to $\mathrm{SU}(6)$ symmetry case labeled "a" and a second with $R_{\omega \Lambda}=1$ labeled "b," where the symmetry is broken. We note that the values of the couplings and that of $U_{\Lambda}^{N}\left(n_{0}\right)$ in Table III are remarkably similar: $R_{\sigma \Lambda} \simeq 0.62$ for the a models and $R_{\sigma \Lambda} \simeq 0.89$ for the b models, and $U_{\Lambda}^{N}\left(n_{0}\right) \simeq-(30-32) \mathrm{MeV}$ for all the models except two of them. In Fig. 1, the experimental values and the theoretical ones obtained after calibration are plotted for the TM1 model with $R_{\omega \Lambda}=2 / 3$.

\section{B. Double $\Lambda$ hypernuclei}

The value of the coupling constants of the $\Lambda$ to the hidden-strangeness mesons $\sigma^{*}$ and $\phi$ is calibrated using the measured $\Lambda \Lambda$ bond energy of ${ }_{\Lambda \Lambda} \mathrm{He}$. Figure 2 shows for the TM1-a and TM1-b models (with $R_{\sigma \Lambda}$ values adjusted to single $\Lambda$ hypernuclei) lines of constant $\Delta B_{\Lambda \Lambda}$ consistent with the experimental values of the bound energy of ${ }_{\Lambda \Lambda}^{6} \mathrm{He}$, i.e., within the error bars defined in Eqs. (6) and (7). In particular, the continuous lines correspond to the limits of Eq. (7), and the dashed line corresponds to the upper limit of Eq. (6), the lower limit being inside the interval defined by Eq. (7). The color contours for Figs. 2(a) and 2(c) indicate the value of the $\Lambda$ potential in $\Lambda$ matter at saturation, $U_{\Lambda}^{(\Lambda)}\left(n_{0}\right)$, obtained from Eq. (11). For completeness, in Figs. 2(b) and 2(d) we also plot contours for $U_{\Lambda}^{(\Lambda)}$ at $n_{0} / 5$ as this is the quantity that has been used to determine the couplings, e.g., in Ref. [18]. The horizontal line corresponds to the SU(6) value $R_{\phi \Lambda}=-\sqrt{2} / 3$. We note that for most values of the ratios $R_{\sigma^{*} \Lambda}=g_{\sigma^{*} \Lambda} / g_{\sigma N}$ and $R_{\phi \Lambda}$, consistent with the experimental constraint from ${ }_{\Lambda \Lambda}^{6} \mathrm{He}$; the value of $U_{\Lambda}^{(\Lambda)}\left(n_{0}\right)$ potential, however, greatly varies and is very different from the value of approximately $-5 \mathrm{MeV}$ generally used in the literature to fix these couplings. In Table IV, we indicate the values of $R_{\sigma^{*} \Lambda}, U_{\Lambda}^{(\Lambda)}\left(n_{0}\right)$, and $U_{\Lambda}^{(\Lambda)}\left(n_{0} / 5\right)$ for two choices of the ratio $R_{\phi \Lambda}$ : the one corresponding the SU(6) symmetry, $R_{\phi \Lambda}=-\sqrt{2} / 3 \simeq-0.471$, and another one $R_{\phi \Lambda}=-\sqrt{2} / 2 \simeq$ -0.707 for which the symmetry is broken. The values of $R_{\sigma^{*} \Lambda}$ and $R_{\phi \Lambda}$ have been obtained after calibrating to the lower and upper values of the ${ }_{\Lambda \Lambda}^{6} \mathrm{He}$ binding energy given in Eq. (7), $\Delta B_{\Lambda \Lambda}=0.50$ and $\Delta B_{\Lambda \Lambda}=0.84 \mathrm{MeV}$, respectively, for our set of models. On the one hand, $U_{\Lambda}^{(\Lambda)}$ at saturation is shown to vary from approximately -14 to $-8 \mathrm{MeV}$, taking the $\mathrm{SU}(6)$ values for vector mesons couplings, and -13 and $+9 \mathrm{MeV}$ if the vector meson couplings to hyperons are imposed to be not larger than to nucleons. These ranges strongly differ from the generally employed one in the literature, i.e., between -1 and $-5 \mathrm{MeV}$, showing that the use of such values for $U_{\Lambda}^{(\Lambda)}$ is inconsistent with the hypernuclei data. On the other hand, the values of $U_{\Lambda}^{(\Lambda)}$ evaluated at $n_{0} / 5$ are restricted to a smaller range: -5 to $-11 \mathrm{MeV}$ approximately. On the whole, Tables III and IV provide the complete set of values of the coupling constants for the $\Lambda$ calibrated to hypernuclear data for all our parametrizations. 

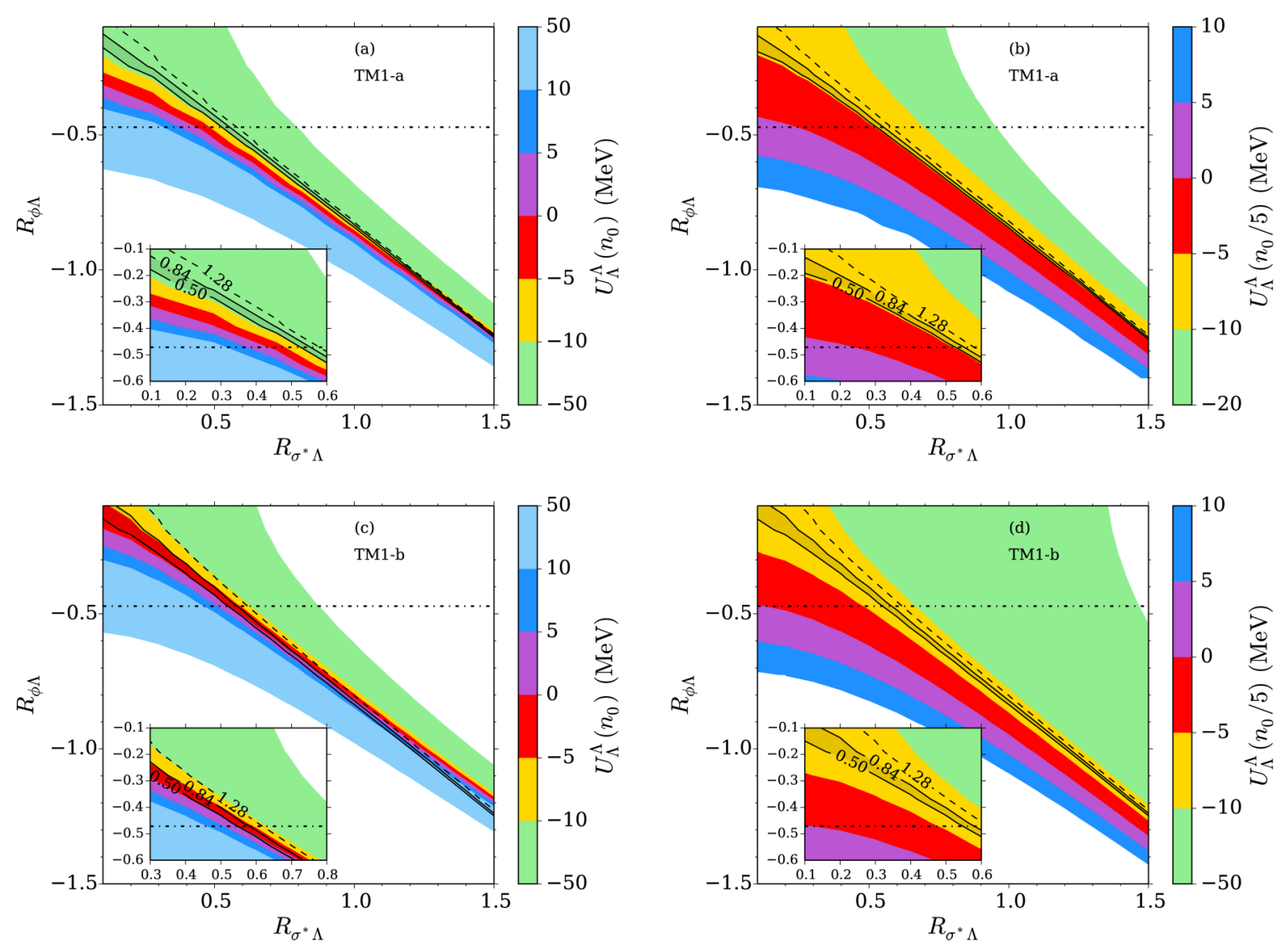

FIG. 2. TM1-a (top) and TM1-b (bottom) models. The solid and dashed black lines correspond to values of $\left(R_{\sigma^{*} \Lambda}, R_{\phi \Lambda}\right)$ consistent with the experimental values of the bound energy of ${ }_{\Lambda \Lambda}^{6} \mathrm{He}$ in Eqs. (6) and (7). Color contours: values of $U_{\Lambda}^{\Lambda}$ at (a) and (c) saturation density $n_{0}$ and (b) and (d) $n_{0} / 5$ obtained from Eq. (11).

\section{HYPERONIC NEUTRON STARS}

We now explore how the calibration of the coupling constants for the $\Lambda$ hyperon to the binding energies of single and double $\Lambda$ hypernuclei affects the properties of neutron stars, in particular the maximum mass. To do so, we calculate the EoS for neutron star matter. Following the work of two of the authors [52], unified EoS are built. For the outer crust, we take the EoS proposed in Ref. [104], for the inner crust we perform a Thomas-Fermi calculation and allow for nonspherical clusters according to Refs. [105,106], and for the core we consider the homogeneous matter EoS.

It is well known that the consequence of the inclusion of hyperons is a softening of the EOS and thus a reduction of its maximum mass $M_{\max }$ compared to the purely nucleonic case. The more hyperonic species at the density corresponding to the central one of the NS with the maximum mass, the smaller the value of $M_{\max }$. Consequently, we consider two types of hyperonic models for the neutron star core: (i) a model in which in addition to the nucleons only the $\Lambda$ hyperon is present, and (ii) a second one where we allow for the appearance of all the hyperon species from the baryonic octet. The first model constitutes a minimal hyperonic model in the sense that only $\Lambda$, if they appear, are present at high density and, therefore, compared to models with a richer hyperonic composition it will predict largest maximum masses. Thus it defines the upper limit on the maximum mass of an hyperonic neutron star.

For the second model, in principle a procedure similar to the one presented in the previous section for $\Lambda$ could be used to determine the couplings for $\Sigma$ and $\Xi$ hyperons with the different mesons. However, as mentioned in Sec. III, there is not yet an unambiguous experimental confirmation of the existence of the $\Sigma$ hypernuclei and very few $\Xi$ hypernuclei have been observed. Hence, the couplings of the $\sigma$ meson to the $\Xi$ and $\Sigma$ hyperons cannot be calibrated using hypernuclear data. Therefore, in this case, we fix the value of the singleparticle potentials of the $\Sigma$ and $\Xi$ and use equations equivalent to Eq. (10) to determine these couplings. In order to explore the dependence of the neutron star maximum mass on the choice of potentials, we choose a repulsive potential for the $\Sigma$ hyperons: $U_{\Sigma}^{N}\left(n_{0}\right)=0,+30 \mathrm{MeV}$ and $U_{\Xi}^{N}\left(n_{0}\right)=-14 \mathrm{MeV}$ or $U_{\Xi}^{N}\left(2 n_{0} / 3\right)=-14 \mathrm{MeV}$ as suggested by the observations of $\Xi$ hypernuclei [71,103]. In addition, since double $\Xi$ or double $\Sigma$ hypernuclei have not been observed, in this work we do not include the coupling of these hyperons with the $\phi$ and $\sigma^{*}$ mesons. We adopt the SU(6) values for the couplings to vector-isoscalar mesons:

$$
\begin{aligned}
& g_{\omega \Xi}=\frac{1}{3} g_{\omega N}=\frac{1}{2} g_{\omega \Sigma}, \\
& g_{\phi \Xi}=2 g_{\phi \Sigma}=-\frac{2 \sqrt{2}}{3} g_{\omega N}
\end{aligned}
$$


TABLE IV. Calibration to double $\Lambda$ hypernuclei for all models a and b. For given $R_{\phi \Lambda}, R_{\sigma^{*} \Lambda}$ are calibrated to reproduce the upper and lower values of bound energy of ${ }_{\Lambda \Lambda}^{6} \mathrm{He}$. For reference, the $\Lambda$ potential in pure $\Lambda$ matter at saturation and at $n_{0} / 5$ are also given. All energies are given in $\mathrm{MeV}$.

\begin{tabular}{|c|c|c|c|c|c|c|c|}
\hline \multirow[t]{2}{*}{ Model } & \multirow[b]{2}{*}{$R_{\phi \Lambda}$} & \multicolumn{3}{|c|}{$\Delta B_{\Lambda \Lambda}=0.50$} & \multicolumn{3}{|c|}{$\Delta B_{\Lambda \Lambda}=0.84$} \\
\hline & & $R_{\sigma^{*} \Lambda}$ & $U_{\Lambda}^{\Lambda}\left(n_{0}\right)$ & $U_{\Lambda}^{\Lambda}\left(n_{0} / 5\right)$ & $R_{\sigma^{*} \Lambda}$ & $U_{\Lambda}^{\Lambda}\left(n_{0}\right)$ & $U_{\Lambda}^{\Lambda}\left(n_{0} / 5\right)$ \\
\hline \multirow[t]{2}{*}{ TM1-a } & $-\sqrt{2} / 3$ & 0.533 & -11.2 & -5.3 & 0.557 & -14.2 & -5.9 \\
\hline & $-\sqrt{2} / 2$ & 0.833 & -10.0 & -5.4 & 0.849 & -13.0 & -6.0 \\
\hline \multirow[t]{2}{*}{ TM1-b } & $-\sqrt{2} / 3$ & 0.549 & -0.2 & -6.8 & 0.580 & -4.1 & -7.6 \\
\hline & $-\sqrt{2} / 2$ & 0.843 & 2.7 & -6.8 & 0.864 & -1.2 & -7.7 \\
\hline \multirow[t]{2}{*}{$\mathrm{TM} 2 \omega \rho-\mathrm{a}$} & $-\sqrt{2} / 3$ & 0.529 & -11.3 & -5.4 & 0.553 & -14.2 & -6.0 \\
\hline & $-\sqrt{2} / 2$ & 0.828 & -10.1 & -5.5 & 0.844 & -13.0 & -6.1 \\
\hline \multirow[t]{2}{*}{$\mathrm{TM} 2 \omega \rho-\mathrm{b}$} & $-\sqrt{2} / 3$ & 0.545 & 0.9 & -6.9 & 0.577 & -3.1 & -7.7 \\
\hline & $-\sqrt{2} / 2$ & 0.838 & 3.6 & -7.0 & 0.859 & -0.2 & -7.8 \\
\hline \multirow[t]{2}{*}{ NL3-a } & $-\sqrt{2} / 3$ & 0.534 & -9.9 & -5.6 & 0.559 & -13.2 & -6.3 \\
\hline & $-\sqrt{2} / 2$ & 0.835 & -8.4 & -5.7 & 0.851 & -11.6 & -6.4 \\
\hline \multirow[t]{2}{*}{ NL3-b } & $-\sqrt{2} / 3$ & 0.552 & 5.2 & -7.0 & 0.586 & 0.8 & -8.0 \\
\hline & $-\sqrt{2} / 2$ & 0.846 & 9.0 & -7.1 & 0.868 & 4.8 & -8.0 \\
\hline \multirow[t]{2}{*}{$\mathrm{NL} 3 \omega \rho-\mathrm{a}$} & $-\sqrt{2} / 3$ & 0.534 & -9.4 & -5.5 & 0.560 & -12.8 & -6.2 \\
\hline & $-\sqrt{2} / 2$ & 0.835 & -7.9 & -5.6 & 0.851 & -11.2 & -6.3 \\
\hline \multirow[t]{2}{*}{$\mathrm{NL} 3 \omega \rho-\mathrm{b}$} & $-\sqrt{2} / 3$ & 0.552 & 5.2 & -7.0 & 0.586 & 0.8 & -8.0 \\
\hline & $-\sqrt{2} / 2$ & 0.846 & 9.0 & -7.1 & 0.868 & 4.8 & -8.0 \\
\hline \multirow[t]{2}{*}{ DDME2-a } & $-\sqrt{2} / 3$ & 0.538 & -8.4 & -2.7 & 0.561 & -11.6 & -3.4 \\
\hline & $-\sqrt{2} / 2$ & 0.828 & -6.2 & -2.7 & 0.843 & -9.4 & -3.4 \\
\hline \multirow[t]{2}{*}{ DDME2-b } & $-\sqrt{2} / 3$ & 0.563 & 1.6 & -2.8 & 0.592 & -2.5 & -3.7 \\
\hline & $-\sqrt{2} / 2$ & 0.844 & 6.6 & -2.8 & 0.864 & 2.6 & -3.7 \\
\hline \multirow[t]{2}{*}{ DDME2D-a } & $-\sqrt{2} / 3$ & 0.535 & -11.9 & -4.1 & 0.555 & -11.7 & -4.0 \\
\hline & $-\sqrt{2} / 2$ & 0.826 & -10.6 & -4.0 & 0.840 & -10.6 & -4.0 \\
\hline \multirow[t]{2}{*}{ DDME2D-b } & $-\sqrt{2} / 3$ & 0.564 & -6.7 & -4.3 & 0.588 & -6.6 & -4.3 \\
\hline & $-\sqrt{2} / 2$ & 0.846 & -3.4 & -4.3 & 0.862 & -3.4 & -4.3 \\
\hline
\end{tabular}

and assume

$$
g_{\rho \Xi}=\frac{1}{2} g_{\rho \Sigma}=g_{\rho N}
$$

for the $\rho$ meson, taking into account the isospin properties of the different baryons.

Here we should mention that the authors of Refs. [20,107] have considered the SU(3) flavor symmetric model to fix the couplings of the hyperons to the three mesons, $\sigma, \omega$, and $\rho$, and have obtained for the last two the same couplings we define in Eqs. (16)-(18). For the $\sigma$-hyperon couplings, they arrive at the equality

$$
2\left(g_{N \sigma}+g_{\Xi \sigma}\right)=3 g_{\Lambda \sigma}+g_{\Sigma \sigma},
$$

which they complement with two extra conditions: that the hyperon couplings are positive and smaller than the nucleon ones [107]. From $\Lambda$ hypernuclei, the ratio $R_{\sigma \Lambda}$ was fixed to 0.616 [20] for the DDME2 model. This value of $R_{\sigma \Lambda}$ together with the relation (19) and the condition $0 \leqslant g_{\Xi \sigma} \leqslant g_{N \sigma}$ results in the following range of values for $R_{\sigma \Sigma}: 0.15 \leqslant R_{\sigma \Sigma} \leqslant 0.45$. Using these values for the meson-hyperon coupling ratios, one can determine the hyperonic potentials in symmetric nuclear matter, taking the hyperon coupling parameters constant. Although not indicated, this seems to have been the choice in Refs. [20,107] since Eq. (33) in Ref. [107] applies to
TABLE V. Hyperon- $\sigma$ coupling ratios and hyperonic potentials in symmetric nuclear matter at saturation, using the conditions defined in Refs. [20,107].

\begin{tabular}{lccccc}
\hline \hline$R_{\sigma \Lambda}$ & $R_{\sigma \Sigma}$ & $R_{\sigma \Xi}$ & $\begin{array}{c}U_{\Lambda} \\
(\mathrm{MeV})\end{array}$ & $\begin{array}{c}U_{\Sigma} \\
\mathrm{MeV})\end{array}$ & $\begin{array}{c}U_{\Xi} \\
(\mathrm{MeV})\end{array}$ \\
\hline 0.6164 & 0.15 & 0 & -32.6 & 154.9 & 107.7 \\
0.6164 & 0.45 & 0.15 & -32.6 & 34.3 & 47.5 \\
0.6164 & 0.76 & 0.30 & -32.6 & -88.6 & -14.0 \\
\hline \hline
\end{tabular}

constant couplings. The results are shown in Table V. The first two lines of this table have been obtained by taking the lower and upper values for the ratio $R_{\sigma \Sigma}$. While the value of $U_{\Lambda}\left(n_{0}\right)$ is within the expected range since $R_{\sigma \Lambda}$ was fitted to the $\Lambda$ hypernuclei properties, the $\Xi$ potential is very repulsive, contrary to the experimental results which seem to indicate that approximately $-14 \mathrm{MeV}$ would be a reasonable value [71,103]. Keeping now the $R_{\sigma \Lambda}$ ratio and choosing $R_{\sigma \Xi}$ such that $U_{\Xi}\left(n_{0}\right)=-14 \mathrm{MeV}$, Eq. (19) can be used to determine $R_{\sigma \Sigma}$. The results are shown in the last line of Table V. One immediately sees that the $\Sigma$ potential comes out very attractive when in fact it is expected to be repulsive. It appears therefore that the constraints resulting from the SU(3) flavor symmetric model for the hyperon-scalar-meson coupling constants are not compatible with simultaneous attractive $\Xi$ potential and repulsive $\Sigma$ potential, and thus are in contradiction with what experiments seem to indicate, as discussed in Sec. III.

Figure 3(a) shows the maximum mass $M_{\max }$ obtained when solving the Tolman-Oppenheimer-Volkoff (TOV) equations [108] for an EoS based on the TM1-a parametrization as a function of $R_{\phi \Lambda}$ for the two types of hyperonic models mentioned before, both with $\Lambda$ couplings adjusted to single and double $\Lambda$ hypernuclei. In addition, the horizontal gray line indicates the maximum mass obtained for a purely nucleonic core $M_{\max }^{\mathrm{N}}$ (see Table II) and the arrow shows the value of $R_{\phi \Lambda}$ corresponding to SU(6) symmetry. Figure 3(b) shows, for the TM1-a model and the two values of the $U_{\mathrm{\Xi}}^{N}$ potential, the composition inside the core of a neutron star with a mass equal to $M_{\max }$, taking $R_{\phi \Lambda}$ equal to its $\mathrm{SU}(6)$ value and $\Lambda$ couplings adjusted to $\Delta B_{\Lambda \Lambda}=0.50 \mathrm{MeV}$ and to single hypernuclei.

The influence of the value of the potential for the $\Sigma$ hyperons on the maximum mass is found to be small. Indeed, the $\Xi$ are, after the $\Lambda$, the most numerous hyperons, owing to the fact that the $\Sigma$ potential is repulsive, and the fraction of $\Sigma$, even if they appear, is approximately one order of magnitude smaller, as shown in Fig. 3(b). Similarly, the value of the bound energy of ${ }_{\Lambda \Lambda}^{6} \mathrm{He}$ hardly affects the results since very similar values of $R_{\sigma^{*} \Lambda}$ are obtained for $\Delta B_{\Lambda \Lambda}=0.50$ or $0.84 \mathrm{MeV}$ as indicated in Table IV. The lower bound for TM1-b is very flat, showing no dependence on $R_{\phi \Lambda}$ because the $\Lambda$ hyperons are suppressed and if present they only appear in residual quantities. As an example, see Fig. 7(b) where a similar choice of couplings for $\Lambda$ is considered.

The second type of hyperonic model is a maximal hyperonic model, which sets a lower limit on the neutron star maximum mass with an hyperonic EoS, since for the $\Sigma$ and $\Xi$ hyperons the inclusion of the vector $\phi$ meson will bring extra repulsion 

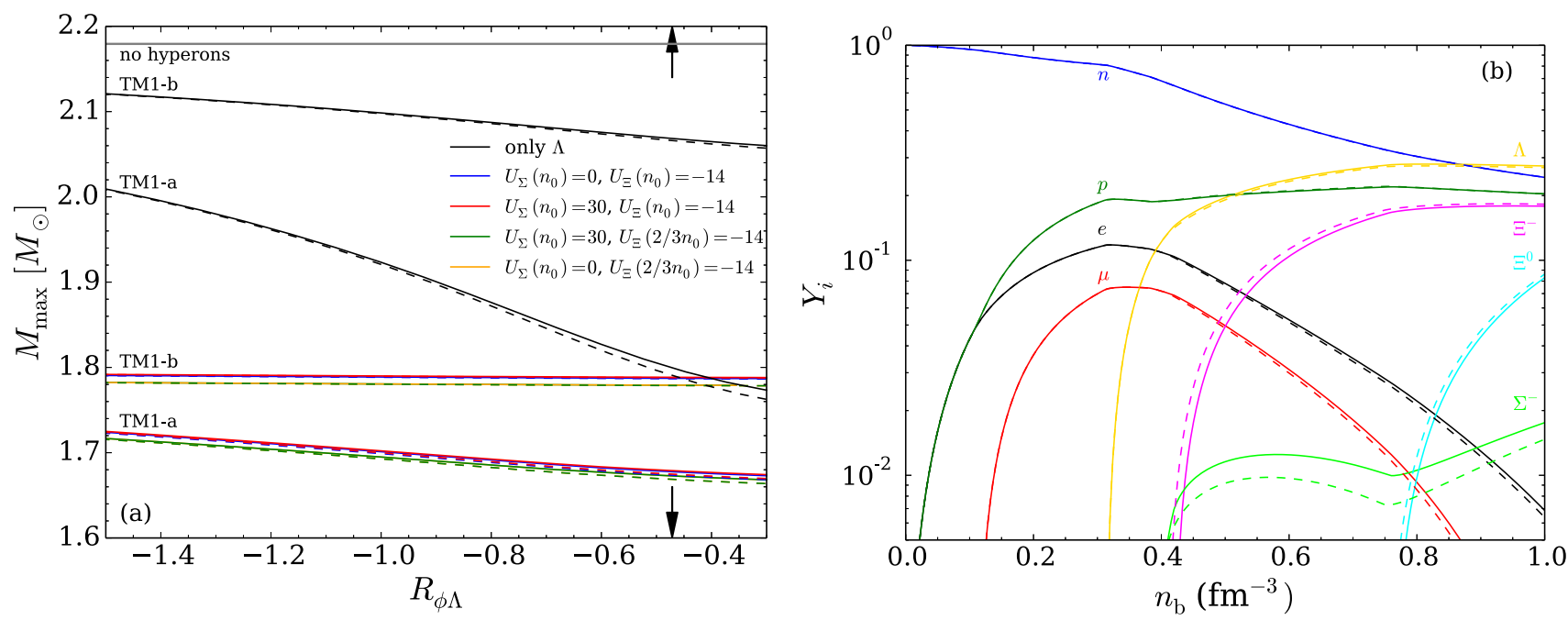

FIG. 3. (a) Neutron star maximum mass $M_{\max }$ as a function of $R_{\phi \Lambda}$ for for the TM1-a and TM1-b models and various hyperonic models. The values $R_{\sigma \Lambda}, R_{\phi \Lambda}$, and $R_{\sigma^{*} \Lambda}$ are adjusted to reproduce the binding energies of single $\Lambda$ hypernuclei and of ${ }_{\Lambda \Lambda}^{6} \mathrm{He}$ with $\Delta B_{\Lambda \Lambda}=0.50 \mathrm{MeV}$ (solid lines) and $0.84 \mathrm{MeV}$ (dashed lines). The dotted line indicates the $\mathrm{SU}(6)$ value of $R_{\phi \Lambda}$; (b) composition of the neutron star core for the TM1-a model and $R_{\phi \Lambda}$ equal to its $\mathrm{SU}(6)$ value and $R_{\sigma \Lambda}, R_{\sigma^{*} \Lambda}$ calibrated to hypernuclei data with $\Delta B_{\Lambda \Lambda}=0.50 \mathrm{MeV}$, for $U_{\Sigma}\left(n_{0}\right)=0 \mathrm{MeV}$ and $U_{\Xi}\left(n_{0}\right)=-14$ (solid lines) or $U_{\Xi}\left(2 / 3 n_{0}\right)=-14 \mathrm{MeV}$ (dashed lines).

even if the scalar $\sigma^{*}$ meson is also included, due to the vector dominance at high densities. Consequently, with the two types of hyperonic models we can calculate the range of neutron star maximum masses consistent with the available experimental data on hypernuclei and compare it to the astrophysical constraints on $M_{\max }$. The width of this $M_{\max }$ range reflects our current uncertainty or lack of information on the $Y N$ and $Y Y$ interactions.

For the TM1-a model, as shown in Fig. $3,2 \mathrm{M}_{\odot}$ can only be reached when $R_{\phi \Lambda}<-1.5$, i.e., when the $\mathrm{SU}(6)$ is very strongly broken, and with the condition that only $\Lambda$ hyperons are included in the model. Therefore, this model appears to be difficult to reconcile with both astrophysical and hypernuclear data. In contrast, as far as the TM1-b model is concerned, for any value of $R_{\phi \Lambda}$, the maximal hyperonic model gives $M_{\max }>1.78 \mathrm{M}_{\odot}$ and the minimum one gives $M_{\max }<2.06-2.12 \mathrm{M}_{\odot}$. Thus, hyperonic EoS consistent with a maximum mass of $2 \mathrm{M}_{\odot}$ and current hypernuclear data can be obtained.

A similar approach is used for the four additional parametrizations. As shown in Fig. 4 for TM $2 \omega \rho$-a model, a maximum mass of $2 \mathrm{M}_{\odot}$ is reached if $R_{\sigma^{*} \Lambda} \leqslant-1$ for the minimal hyperonic model. Again, as for TM1, the breaking $\mathrm{SU}(6)$ symmetry is required, but to a lesser extent since the maximum masses are larger for the TM $2 \omega \rho$ parametrization than for TM1 one. For the TM $2 \omega \rho$-b model, the maximum mass reachable for the minimal hyperonic model is always larger than $2 \mathrm{M}_{\odot}: M_{\max }>2.16-2.19 \mathrm{M}_{\odot}$. This model is thus compatible with both hypernuclear and astrophysical data.

Very similar results are obtained when comparing the NL3 and NL3 $\omega \rho$ parametrizations, shown in Fig. 5. It has to be mentioned that for models with $R_{\omega \Lambda}=2 / 3$ and for $R_{\phi \Lambda}$ small in absolute value maximum mass, but when the baryon effective mass becomes equal to 0 . Such cases are not plotted and no acceptable solutions were found, because the effective mass of the nucleons becomes negative at densities that occur inside a neutron star. The curves plotted in Fig. 5 correspond to the hyperon-meson parametrizations which lead to physically acceptable results, in particular, a positive effective mass. This explains why the curves for the NL3-a and NL3 $\omega \rho$-a models extend only until -0.7 and from -0.9 to -0.8 , respectively, contrary to the curves of the other models shown in Figs. 3, 4 , and 6 , which extend until $-\sqrt{2} / 3$. Any model for the NL3 and NL3 $\omega \rho$ parametrizations is consistent with the existence of a $2 \mathrm{M}_{\odot}$ neutron star and they even predict the possibility of having hyperonic neutron stars with masses at least larger than $2.1 \mathrm{M}_{\odot}$.

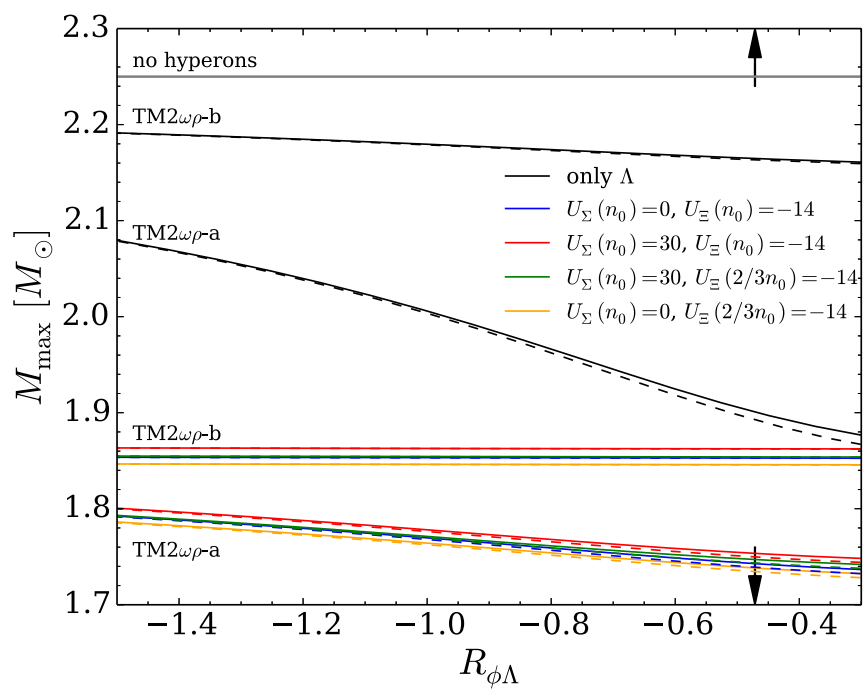

FIG. 4. Analog of Fig. 3(a) for the TM2 $\omega \rho$ parametrization. 

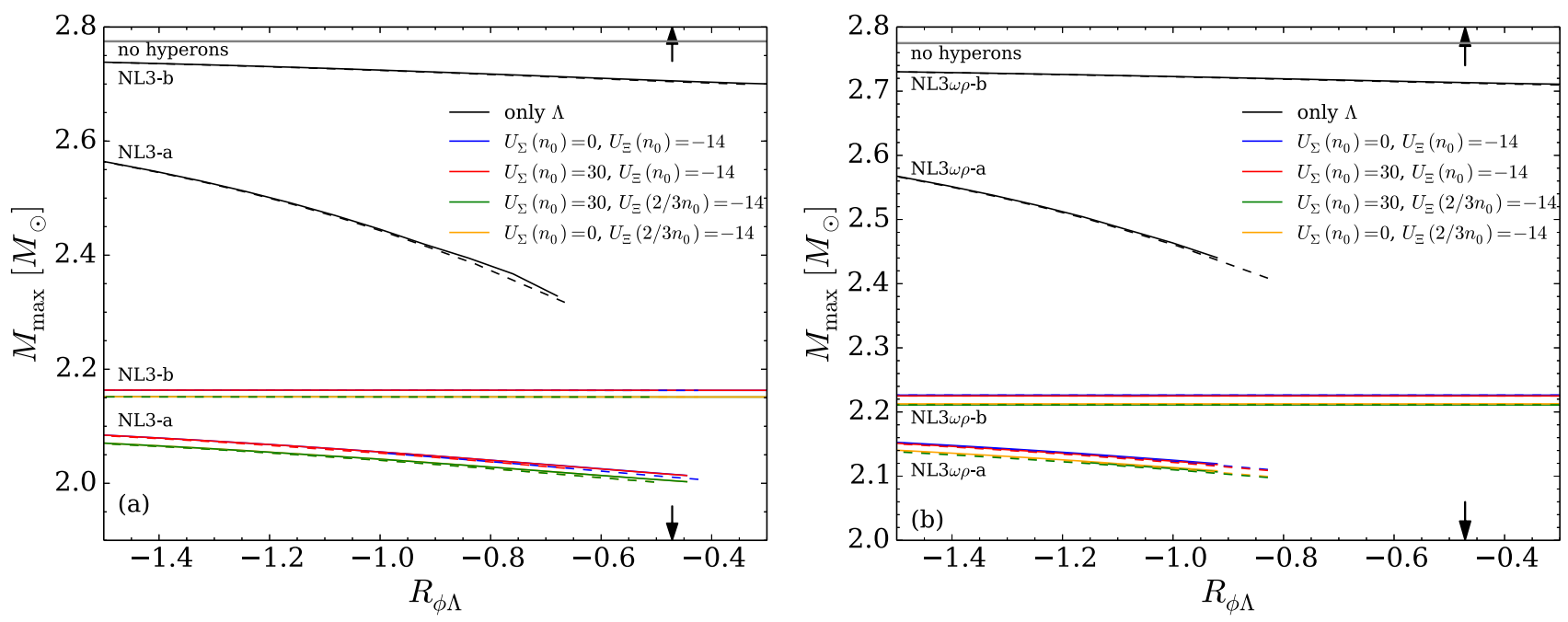

FIG. 5. Analog of Fig. 3(a) for the (a) NL3 and (b) NL3 $\omega \rho$ parametrizations.

Results for the DDME2 and DDME2D parametrizations are plotted in Fig. 6. Whether hyperon couplings are density dependent or not appears not to affect the maximum mass for the models $a$, whereas it does for the models $b$.

For the DDME2 parametrization, Fig. 7 shows that for model a the $\Lambda$ is the first hyperon to set in. For a density slightly larger, $\Sigma^{-}$also appears and the fraction of electrons and $\mu$ decreases. However, as soon $\Xi^{-}$sets in, it is favored because the repulsive $g_{\omega \Xi}$ coupling is one half of the coupling of the $\omega$ to the $\Sigma^{-}$. Taking $g_{\omega \Lambda}=1$, the $\Lambda$ hyperon becomes disfavored and only a small fraction at quite high densities appears in the case with only nucleons and $\Lambda$ 's. If the other hyperons are also taken into account, there are no $\Lambda$ 's below $n_{\mathrm{b}}=1 \mathrm{fm}^{-3}$.

For the DDME2D parametrization, the hyperon-meson couplings are weaker than in the previous scenario, and they decrease with the density as the nucleon-meson couplings. A weaker $g_{\omega \Sigma}$ allows a lower onset density, and since in this model the $\rho$-meson coupling is quite strong, the $\Sigma^{-}$sets in first, as shown in Fig. 8 . The $\Lambda$ hyperon sets in at a density very close to $\Sigma^{-}$if $R_{\omega \Lambda}=2 / 3$; otherwise if $R_{\omega \Lambda}=1$ its onset is shifted to quite high densities and its fraction is always below $1 \%$. As in the case with constant couplings, as soon as the $\Xi^{-}$sets, in the amount of the $\Sigma^{-}$decreases steadily, since $R_{\omega \Xi}=1 / 3$ is half the corresponding coupling for the $\Sigma^{-}$. Having more strict constraints to fix the different hyperon-meson couplings and including the strangeness hidden mesons, $\sigma^{*}$ and $\phi$, the relative abundances will certainly change, but the total amount of strangeness is less sensitive to the relative magnitude of the couplings. For instance, making the $\Sigma$ potential in nuclear matter repulsive will certainly reduce the amount of $\Sigma^{-}$ present in matter, increasing the amount of the other hyperons.
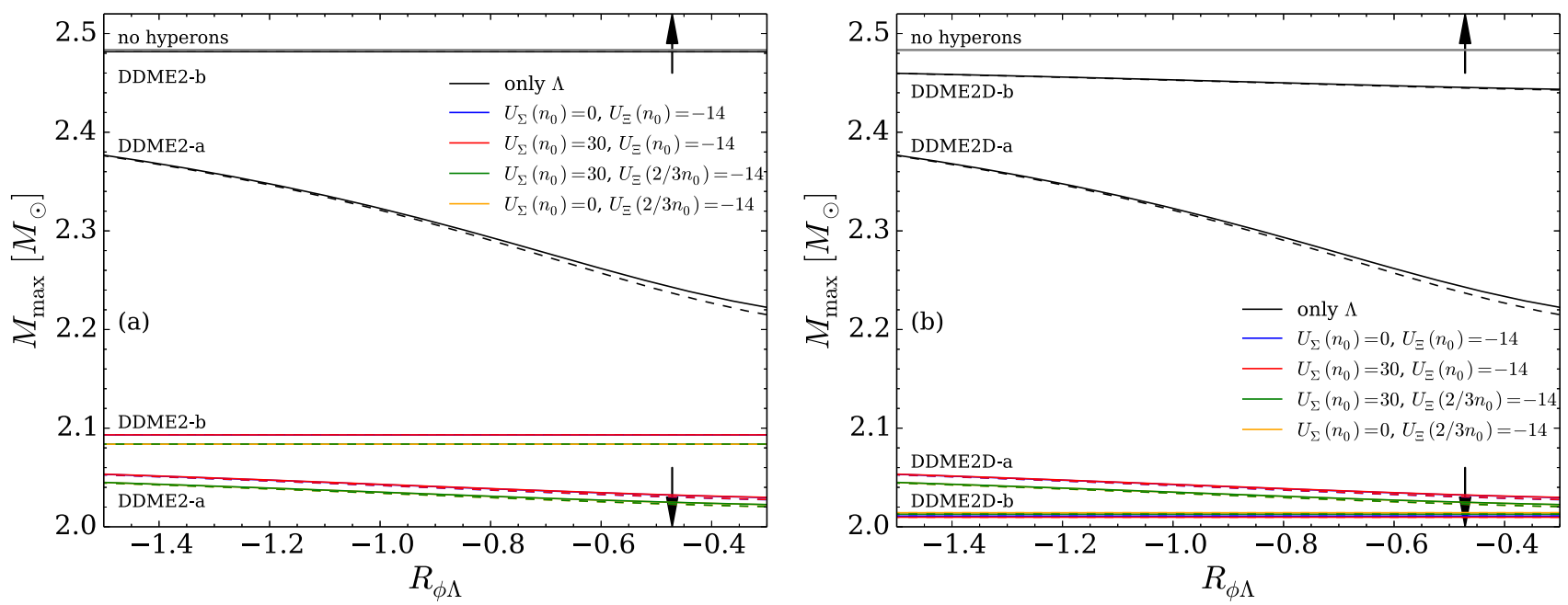

FIG. 6. Analog of Fig. 3 for the (a) DDME2 and (b) DDME2D parametrizations. 

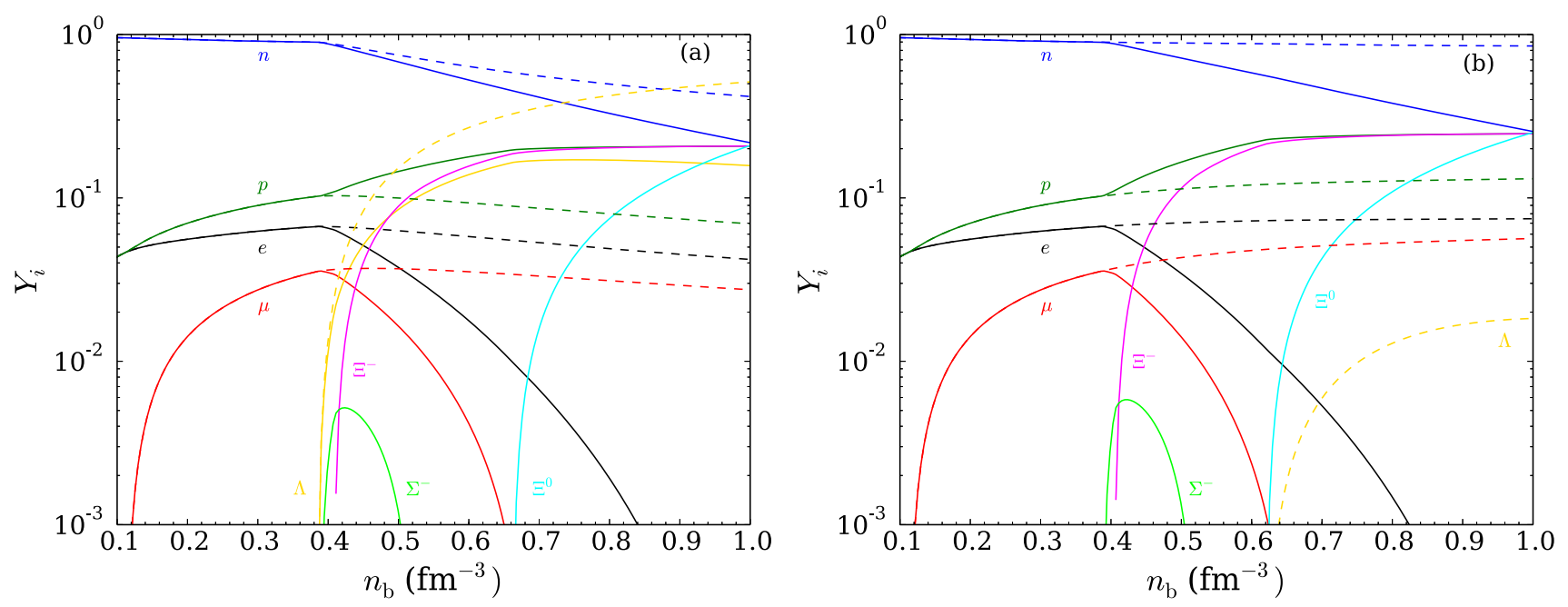

FIG. 7. DDME2 parametrization with (a) SU(6), i.e., $R_{\omega \Lambda}=2 / 3$ and (b) $R_{\omega \Lambda}=1$ obtained for $R_{\phi \Lambda}$ equal to its $\mathrm{SU}(6)$ value and $R_{\sigma \Lambda}$ and $R_{\sigma^{*} \Lambda}$ calibrated to hypernuclear data with $\Delta B_{\Lambda \Lambda}=0.5 \mathrm{MeV}$. Dashed lines: only $\Lambda$ s included; solid lines: all hyperons included with $U_{\Sigma}=0$ and $U_{\Xi}\left(n_{0}\right)=-14 \mathrm{MeV}$.

\section{SUMMARY AND CONCLUSIONS}

In this work, we explore whether phenomenological RMF models of the hyperonic matter EoS fitted to up-to-date hypernuclei data are consistent with the existence of $2 \mathrm{M}_{\odot}$ neutron stars.

Modeling single and double $\Lambda$ hypernuclei, we first calibrate the $\Lambda$ couplings for six different RMF parametrizations. The usual way of calibrating the $R_{\sigma \Lambda}$ coupling by imposing the value of the $\Lambda$ potential in symmetric baryonic matter, i.e., using $U_{\Lambda}^{N}\left(n_{0}\right) \simeq-30 \mathrm{MeV}$, appears in agreement with the binding energy of single $\Lambda$ hypernuclei in the $s$ and $p$ shells. Moreover, the value of $R_{\sigma \Lambda}$ that comes out of the order of $\sim 0.62$ when the $\mathrm{SU}(6)$ value for $g_{\omega \Lambda}$ is taken is quite independent of the model considered. This is not at all the case for the calibration of the $R_{\sigma^{*} \Lambda}$ and $R_{\phi \Lambda}$ couplings. Calibrating to the bound energy ${ }_{\Lambda \Lambda}^{6} \mathrm{He}$ shows that for the models $\mathrm{a}$ and $\mathrm{b}$ the $\Lambda$ potential in pure $\Lambda$ matter $U_{\Lambda}^{\Lambda}\left(n_{0}\right)$

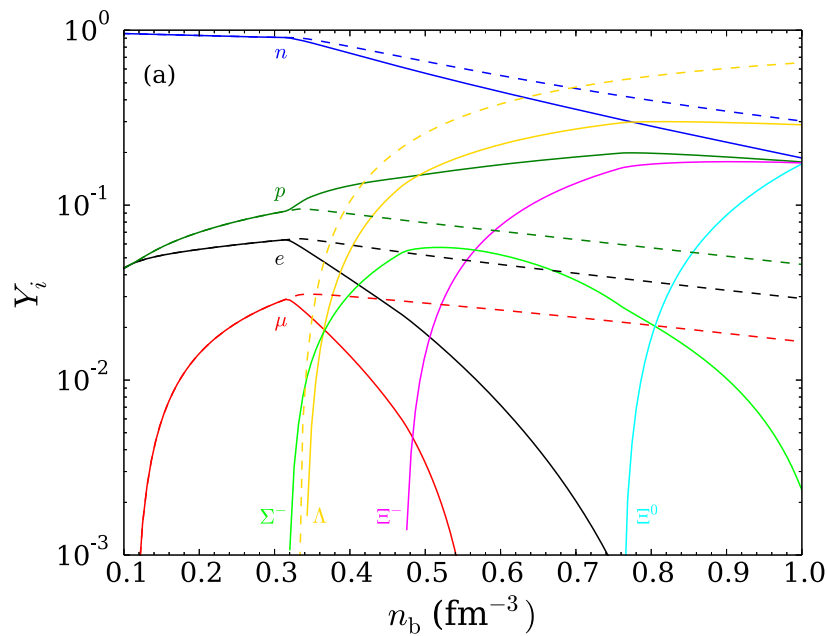

varies between approximately -14 and $+9 \mathrm{MeV}$. This is at variance with the usual values of -1 or $-5 \mathrm{MeV}$ employed in the literature, showing that these values are inconsistent with the hypernuclear data. Tables III and III provide the values of the various couplings to the $\Lambda$ calibrated to hypernuclear data.

We then proceed by constructing unified hyperonic EoS for neutron star matter. While an approach similar to the one presented for the $\Lambda$ hyperon should in principle be used for the $\Sigma$ and $\Xi$ ones, the lack of hypernuclear experimental data does not allow us to calibrate their couplings to hypernuclei properties. Consequently, we proceed by devising two limiting hyperonic models. In the minimal one, only the $\Lambda$ hyperon is included in addition to the nucleons and its couplings are calibrated to hypernuclear data. The $\Sigma$ and $\Xi$ hyperons are included in the maximal hyperonic model using a repulsive potential in symmetric baryonic matter for the $\Sigma$ and a value for $\Xi$ of $-14 \mathrm{MeV}$, consistent with the scarce experimental

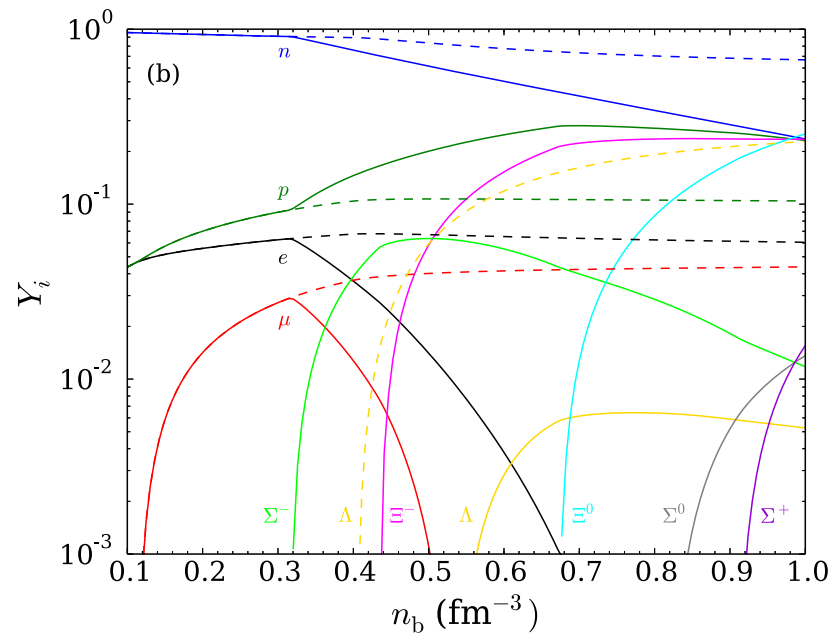

FIG. 8. Same as Fig. 7 for DDME2D parametrization. 
constraint for this hyperon. For these two hyperons, no coupling to the hidden mesons $\sigma^{*}$ and $\phi$ is included because of the nonexisting experimental data that would allow us to constrain the coupling parameters.

Finally, we confront the EoS calibrated to hypernuclear data to the astrophysical constraint that neutron stars with $2 \mathrm{M}_{\odot}$ exist. For the TM1 and TM $2 \omega \rho$ parametrizations, the breaking of the SU(6) symmetry appears required to be consistent with this constraint, and it is still not clear if even breaking the SU(6) symmetry the $2 \mathrm{M}_{\odot}$ limit is satisfied when all the hyperons of the baryonic octet also interact with the $\sigma^{*}$ and $\phi$ mesons. The NL3, NL3 $\omega \rho$ models predict the existence of hyperonic stars with masses larger than at least $2 \mathrm{M}_{\odot}$. However, these two models may pose some problems when hyperons are included, depending on the value of the hyperon-meson coupling: For some choices of the couplings, the effective mass of the nucleons becomes negative at densities that occur inside a neutron star. As a consequence, these models are unacceptable if future constraints indicate that the appropriate couplings lie outside their physically acceptable range. The DDME2 and DDME2D models are both consistent with the $2 \mathrm{M}_{\odot}$ constraint. In addition, two of the models, NL3 and TM1, are, according to experimental and $a b$ initio neutron matter calculations, excluded due to their large symmetry energy and slope [51]. Among the corresponding models satisfying those constraints, NL3 $\omega \rho$ and TM $2 \omega \rho$ are able to satisfy the $2 \mathrm{M}_{\odot}$ constraint only in a limited range of values of the hyperonmeson coupling parameters and extra information is needed to decide their validity. Finally, the presently existing information on hypernuclei does not seem to put any constraint on DDME2 and DDME2D. In summary, up-to-date hypernuclei data set some strong restrictions on the RMF parametrizations with constant couplings and acceptable values of the symmetry energy and its slope used in this work, but do not exclude any of the considered models with density-dependent couplings, on the ground that hyperonic stars are not consistent with the existence of $2 \mathrm{M}_{\odot}$ neutron stars.

The properties of the nucleonic sector are themselves hardly constrained at high density. Future measurements of neutron star properties (mass and radius, surface gravitational redshift, moment of inertia, etc.) and of high-density properties of asymmetric nuclear matter in the laboratory appear necessary to constrain further the nucleonic EoS. If in addition properties of $\Xi$ and $\Sigma$ hyperons are better constrained, one could reduce the range of possible maximum masses given by the minimal and maximal hyperonic models and potentially solve the hyperon puzzle.

\section{ACKNOWLEDGMENTS}

Partial support comes from NewCompStar, COST Action MP1304. The work of M.F. has been partially supported by the NCN (Poland) Grant No. 2014/13/B/ST9/02621 and by the STSM grant from the COST Action MP1304, and by Fundação para a Ciência e Tecnologia (FCT), Portugal, under Project No. UID/FIS/04564/2016.
[1] V. A. Ambartsumyan and G. S. Saakyan, Sov. Astron. 4, 187 (1960).

[2] H.-J. Schulze, M. Baldo, U. Lombardo, J. Cugnon, and A. Lejeune, Phys. Lett. B 355, 21 (1995); Phys. Rev. C 57, 704 (1998); M. Baldo, G. F. Burgio, and H.-J. Schulze, ibid. 58, 3688 (1998); 61, 055801 (2000); I. Vidaña, A. Polls, A. Ramos, M. Hjorth-Jensen, and V. G. J. Stoks, ibid. 61, 025802 (2000); I. Vidaña, A. Polls, A. Ramos, L. Engvik, and M. Hjorth-Jensen, ibid. 62, 035801 (2000); H-J. Schulze, A. Polls, A. Ramos, and I. Vidaña, ibid. 73, 058801 (2006); H.-J. Schulze and T. Rijken, ibid. 84, 035801 (2011).

[3] H. Đapo, B.-J. Schaefer, and J. Wambach, Phys. Rev. C 81, 035803 (2010).

[4] F. Sammarruca, Phys. Rev. C 79, 034301 (2009).

[5] T. Katayama and K. Saito, arXiv:1410.7166; Phys. Lett. B 747, 43 (2015).

[6] D. Lonardoni, F. Pederiva, and S. Gandolfi, Phys. Rev. C 89, 014314 (2014)

[7] N. K. Glendenning, Phys. Lett. B 114, 392 (1982); Astrophys. J. 293, 470 (1985); Z. Phys. A 326, 57 (1987); F. Weber and M. K. Weigel, Nucl. Phys. A 505, 779 (1989); R. Knorren, M. Prakash, and P. J. Ellis, Phys. Rev. C 52, 3470 (1995); H. Huber, F. Weber, M. K. Weigel, and Ch. Schaab, Int. J. Mod. Phys. E 7, 301 (1998).

[8] S. Balberg and A. Gal, Nucl. Phys. A 625, 435 (1997); S. Balberg, I. Lichtenstadt, and G. B. Cook, Astrophys. J. Suppl. Ser. 121, 515 (1999); D. E. Lanskoy and Y. Yamamoto, Phys. Rev. C 55, 2330 (1997); T. Y. Tretyakova and D. E. Lanskoy, Eur. Phys. J. A 5, 391 (1999); J. Cugnon, A. Lejeune, and H.-J. Schulze, Phys. Rev. C 62, 064308 (2000); I. Vidaña, A. Polls, A. Ramos, and H.-J. Schulze, ibid. 64, 044301 (2001); X.-R. Zhou, H.-J. Schulze, H. Sagawa, C.-X. Wu, and E.-G. Zhao, ibid. 76, 034312 (2007); X.-R. Zhou, A. Polls, H.-J. Schulze, and I. Vidaña, ibid. 78, 054306 (2008).

[9] R. A. Hulse and J. H. Taylor, Astrophys. J. Lett. 195, L51 (1975).

[10] I. Bombaci, P. K. Panda, C. Providência, and I. Vidaña, Phys. Rev. D 77, 083002 (2008).

[11] R. Cavagnoli, D. P. Menezes, and C. Providência, Phys. Rev. C 84, 065810 (2011).

[12] P. Demorest et al., Nature (London) 467, 1081 (2010).

[13] E. Fonseca et al., Astrophys. J. 832, 167 (2016).

[14] J. Antoniadis et al., Science 340, 1233232 (2013).

[15] M. Fortin, J. L. Zdunik, P. Haensel, and M. Bejger, Astron. Astrophys. 576, A68 (2015).

[16] I. Bednarek, P. Haensel, J. L. Zdunik, M. Bejger, and R. Mańka, Astron. Astrophys. 543, A157 (2012).

[17] S. Weissenborn, D. Chatterjee, and J. Schaffner-Bielich, Phys. Rev. C 85, 065802 (2012).

[18] M. Oertel, C. Providência, F. Gulminelli, and Ad. R. Raduta, J. Phys. G 42, 075202 (2015).

[19] K. A. Maslov, E. E. Kolomeitsev, and D. N. Voskresensky, Phys. Lett. B 748, 369 (2015).

[20] E. N. E. van Dalen, G. Colucci, and A. Sedrakian, Phys. Lett. B 734, 383 (2014).

[21] S. Gandolfi, J. Carlson, and S. Reddy, Phys. Rev. C 85, 032801 (2012). 
[22] K. Hebeler, J. M. Lattimer, C. J. Pethick, and A. Schwenk, Astrophys. J. 773, 11 (2013).

[23] T. Takatsuka et al., Eur. Phys. J. A 13, 213 (2002); Prog. Theor. Phys. Suppl. 174, 80 (2008).

[24] I. Vidaña, D. Logoteta, C. Providência, A. Polls, and I. Bombaci, Eur. Phys. Lett. 94, 11002 (2011).

[25] Y. Yamamoto, T. Furumoto, N. Yasutake, and Th. A. Rijken, Phys. Rev. C 88, 022801(R) (2013); 90, 045805 (2014).

[26] D. Lonardoni, A. Lovato, S. Gandolfi, and F. Pederiva, Phys. Rev. Lett. 114, 092301 (2015).

[27] F. Özel, D. Psaltis, S. Ransom, P. Demorest, and M. Alford, Astrophys. J. Lett. 724, L199 (2010).

[28] S. Weissenborn, I. Sagert, G. Pagliara, M. Hempel, and J. Schaeffner-Bielich, Astrophys. J. Lett. 740, L14 (2011).

[29] T. Klähn, R. Łastowiecki, and D. Blaschke, Phys. Rev. D 88, 085001 (2013).

[30] L. Bonanno and A. Sedrakian, Astron. Astrophys. 539, A16 (2012).

[31] R. Lastowiecki, D. Blaschke, H. Grigorian, and S. Typel, Acta Phys. Polon. Suppl. 5, 535 (2012).

[32] A. Drago, A. Lavagno, G. Pagliara, and D. Pigato, Phys. Rev. C 90, 065809 (2014); EPJ Web Conf. 95, 01011 (2015).

[33] J. Haidenbauer, U.-G. Meissner, N. Kaiser, and W. Weise, arXiv: 1612.03758

[34] M. C. Miller and F. K. Lamb, Eur. Phys. J. A 52, 63 (2016).

[35] P. Haensel, M. Bejger, M. Fortin, and J. L. Zdunik, Eur. Phys. J. A 52, 59 (2016).

[36] S. Guillot, M. Servillat, N. A. Webb, and R. Rutledge, Astrophys. J. 772, 7 (2013).

[37] S. Guillot and R. Rutledge, Astrophys. J. 796, L3 (2014).

[38] V. Dexheimer, R. Negreiros, and S. Schramm, Phys. Rev. C 91, 055808 (2015).

[39] A. Drago, A. Lavagno, and G. Pagliara, Phys. Rev. D 89, 043014 (2014).

[40] K. C. Gendreau, Z. Arzoumanian, and T. Okajima, Proc. SPIE 8443, 844313 (2012).

[41] C. Motch, J. Wilms, D. Barret et al., arXiv:1306.2334.

[42] M. Feroci, J. W. den Herder, E. Bozzo et al., Proc. SPIE 8443, 84432D (2012).

[43] H. Shen, F. Yang, and H. Toki, Prog. Theor. Phys. 115, 325 (2006).

[44] Y. Sugahara and H. Toki, Nucl. Phys. A 579, 557 (1994).

[45] C. Providência and A. Rabhi, Phys. Rev. C 87, 055801 (2013).

[46] G. A. Lalazissis, J. König, and P. Ring, Phys. Rev. C 55, 540 (1997).

[47] C. J. Horowitz and J. Piekarewicz, Phys. Rev. Lett. 86, 5647 (2001).

[48] G. A. Lalazissis, T. Nikšić, D. Vretenar, and P. Ring, Phys. Rev. C 71, 024312 (2005).

[49] H. Shen, H. Toki, K. Oyamatsu, and K. Sumiyoshi, Nucl. Phys. A 637, 435 (1998).

[50] H. Shen, H. Toki, K. Oyamatsu, and K. Sumiyoshi, Astrophys. J. Suppl. 197, 20 (2011).

[51] M. B. Tsang, J. R. Stone, F. Camera et al., Phys. Rev. C 86, 015803 (2012).

[52] M. Fortin, C. Providência, A. R. Raduta, F. Gulminelli, J. L. Zdunik, P. Haensel, and M. Bejger, Phys. Rev. C 94, 035804 (2016).

[53] P. Danielewicz, R. Lacey, and W. G. Lynch, Science 298, 1592 (2002).
[54] M. Dutra, O. Lourenço, S. S. Avancini, B. V. Carlson, A. Delfino, D. P. Menezes, C. Providencia, S. Typel, and J. R. Stone, Phys. Rev. C 90, 055203 (2014).

[55] N. K. Glendenning and S. A. Moszkowski, Phys. Rev. Lett. 67, 2414 (1991).

[56] J. Schaffner and I. N. Mishustin, Phys. Rev. C 53, 1416 (1996).

[57] R. Engelmann et al., Phys. Lett. 21, 587 (1966).

[58] G. Alexander et al., Phys. Rev. 173, 1452 (1968).

[59] B. Sechi-Zor et al., Phys. Rev. 175, 1735 (1968).

[60] J. A. Kadyk et al., Nucl. Phys. B 27, 13 (1971).

[61] J. Eisele et al., Phys. Lett. B 37, 204 (1971).

[62] M. Danysz and J. Pniewski, Phil. Mag. 44, 348 (1953).

[63] M. Danysz et al., Phys. Rev. Lett. 11, 29 (1963).

[64] M. Danysz et al., Nucl. Phys. A 49, 121 (1963).

[65] R. H. Dalitz, D. H. Davis, P. H. Fowler, A. Montwill, J. Pniewski, and J. A. Zakrewski, Proc. Royal Soc. London, Ser. A 426, 1 (1989).

[66] D. J. Prowse, Phys. Rev. Lett. 17, 782 (1966).

[67] S. Aoki et al., Prog. Theor. Phys. 85, 1287 (1991).

[68] C. B. Dover, D. J. Millener, A. Gal, and D. H. Davis, Phys. Rev. C 44, 1905 (1991).

[69] G. B. Franklin, Nucl. Phys. A 585, 83 (1995).

[70] H. Takahashi et al., Phys. Rev. Lett. 87, 212502 (2001).

[71] P. Khaustov et al. (The AGS E885 Collaboration), Phys. Rev. C 61, 054603 (2000).

[72] K. Nakazawa et al., Prog. Theor. Exp. Phys. 2015, 033 D02 (2015).

[73] R. Bertini et al., Phys. Lett. B 90, 375 (1980).

[74] R. Bertini et al., Phys. Lett. B 136, 29 (1984).

[75] R. Bertini et al., Phys. Lett. B 158, 19 (1985).

[76] H. Piekarz et al., Phys. Lett. B 110, 428 (1982).

[77] T. Yamazaki, T. Ishikawa, K. H. Tanaka, Y. Akiba, M. Iwasaki, S. Ohtake, H. Tamura, M. Nakajima, T. Yamanaka, I. Arai et al., Phys. Rev. Lett. 54, 102 (1985).

[78] L. Tang, E. Hungerford, T. Kishimoto, B. Mayes, L. Pinsky, S. Bart, R. Chrien, P. Pile, R. Sutter, P. Barnes et al., Phys. Rev. C 38, 846 (1988).

[79] S. Bart, R. E. Chrien, W. A. Franklin, T. Fukuda, R. S. Hayano, K. Hicks, E. V. Hungerford, R. Michael, T. Miyachi, T. Nagae et al., Phys. Rev. Lett. 83, 5238 (1999).

[80] R. S. Hayano, T. Ishikawa, M. Iwasaki, H. Outa, E. Takada, and H. Tamura, Phys. Lett. B 231, 355 (1989).

[81] T. Nagae, T. Miyachi, T. Fukuda, H. Outa, T. Tamagawa, J. Nakano, R. S. Hayano, H. Tamura, Y. Shimizu et al., Phys. Rev. Lett. 80, 1605 (1998).

[82] C. B. Dover, D. J. Millener, and A. Gal, Phys. Rep. 184, 1 (1989).

[83] C. J. Batty, E. Friedman, and A. Gal, Phys. Lett. B 335, 273 (1994).

[84] C. J. Batty, E. Friedman, and A. Gal, Prog. Theor. Phys. Suppl. 117, 227 (1994).

[85] C. J. Batty, E. Friedman, and A. Gal, Phys. Rep. 287, 385 (1997).

[86] J. Mareš, E. Friedman, A. Gal, and B. K. Jennings, Nucl. Phys. A 594, 311 (1995).

[87] J. Dąbrowski, Phys. Rev. C 60, 025205 (1999).

[88] H. Noumi, P. K. Saha, D. Abe, S. Ajimura, K. Aoki, H. C. Bhang, T. Endo, Y. Fujii, T. Fukuda, H. C. Guo et al., Phys. Rev. Lett. 89, 072301 (2002); 90, 049902(E) (2003). 
[89] P. K. Saha, H. Noumi, D. Abe, S. Ajimura, K. Aoki, H. C. Bhang, K. Dobashi, T. Endo, Y. Fujii, T. Fukuda et al., Phys. Rev. C 70, 044613 (2004).

[90] T. Harada and Y. Hirabayashi, Nucl. Phys. A 759, 143 (2005).

[91] T. Harada and Y. Hirabayashi, Nucl. Phys. A 767, 206 (2006).

[92] E. V. Hungerford, Prog. Theor. Phys. Suppl. 117, 135 (1994).

[93] S. Bianchin et al., Int. J. Mod. Phys. E 18, 2187 (2009).

[94] C. Rappold et al., Nucl. Phys. A 913, 170 (2013).

[95] O. Hashimoto and H. Tamura, Prog. Part. Nucl. Phys. 57, 564 (2006).

[96] P. M. M. Maessen, T. A. Rijken, and J. J. de Swart, Phys. Rev. C 40, 2226 (1989); T. A. Rijken, V. G. J. Stoks, and Y. Yamamoto, ibid. 59, 21 (1999); V. G. J. Stoks and T. A. Rijken, ibid. 59, 3009 (1999); T. A. Rijken, ibid. 73, 044007 (2006); T. A. Rijken and Y. Yamamoto, ibid. 73, 044008 (2006).

[97] J. K. Ahn, H. Akikawa, S. Aoki et al., Phys. Rev. C 88, 014003 (2013).

[98] Y. Sugahara and H. Toki, Prog. Theor. Phys. 92, 803 (1994).
[99] S. S. Avancini, J. R. Marinelli, D. P. Menezes, M. M. W. Moraes, and A. S. Schneider, Phys. Rev. C 76, 064318 (2007).

[100] Y. K. Gambhir, P. Ring, and A. Thimet, Ann. Phys. 198, 132 (1990).

[101] J. V. Noble, Phys. Lett. B 89, 325 (1980).

[102] M. Chiapparini, A. O. Gattone, and B. K. Jennings, Nucl. Phys. A 529, 589 (1991).

[103] A. Gal, E. V. Hungerford, and D. J. Millener, Rev. Mod. Phys. 88, 035004 (2016).

[104] S. B. Rüster, M. Hempel, and J. Schaffner-Bielich, Phys. Rev. C 73, 035804 (2006).

[105] F. Grill, C. Providência, and S. S. Avancini, Phys. Rev. C 85, 055808 (2012).

[106] F. Grill, H. Pais, C. Providência, I. Vidaña, and S. S. Avancini, Phys. Rev. C 90, 045803 (2014).

[107] G. Colucci and A. Sedrakian, Phys. Rev. C 87, 055806 (2013).

[108] J. R. Oppenheimer and G. M. Volkoff, Phys. Rev. 55, 374 (1939); R. C. Tolman, ibid. 55, 364 (1939). 